\title{
A PRIORI ERROR ESTIMATES FOR THE NUMERICAL SOLUTION OF A COUPLED GEOMECHANICS AND RESERVOIR FLOW MODEL WITH STRESS-DEPENDENT PERMEABILITY
}

\author{
SÍLVIA BARBEIRO AND MARY F. WHEELER
}

\begin{abstract}
In this paper we consider the numerical solution of a coupled geomechanics and a stress-sensitive porous media reservoir flow model. We combine mixed finite elements for Darcy flow and Galerkin finite elements for elasticity. This work focuses on deriving convergence results for the numerical solution of this nonlinear partial differential system. We establish convergence with respect to the $L^{2}$-norm for the pressure and for the average fluid velocity and with respect to the $H^{1}$-norm for the deformation. Estimates respect to the $L^{2}$-norm for mean stress, which is of special importance since it is used in the computation of permeability for poroelasticity, can be derived using the estimates in the $H^{1}$-norm for the deformation. We start by deriving error estimates in a continuous-in-time setting. A cut-off operator is introduced in the numerical scheme in order to derive convergence. The spatial grids for the discrete approximations of the pressure and deformation do not need be the same. Theoretical convergence error estimates in a discrete-in-time setting are also derived in the scope of this investigation. A numerical example supports the convergence results.
\end{abstract}

KEYWORDS: stress-sensitive reservoir problem, poro-elasticity, mixed finite elements, a priori error estimates.

\section{Introduction}

The modeling of coupled mechanics and flow in porous media attracts researchers from different areas and is of great importance in a diverse range of engineering fields. Land subsidence, due to consolidation or compaction, which is often caused by exploitation of subsurface resources, has often been a concern for reservoir engineers. Understanding the effects of groundwater pumping or oil extraction and its impact on the environment has been motivating extensive studies in subsurface flow and geomechanics modeling. Poroelastic models are also used in biomechanics. Another major application arises in sequestration of carbon in saline aquifers. Some of the modern applications of poroelasticity modeling are highlighted in [10].

Received May 19, 2009.

This work was partially supported by the UT Austin | Portugal Program, International Collaboratory for Emerging Technologies (CoLab). 
The most recent mathematical models that we find in specialized engineering literature describe very complex fluid/medium interactions and multiple porous media heterogeneities. Clearly, the reliability of predictions depends how well the models describe the realistic field. For example, in stress-sensitive reservoirs, variation of the effective stress resulting from fluid production may induce deformation of the rocks and cause permeability reduction. This effect may significantly reduced expected productivity ([4], [15]). The correlation of stress induced loss in productivity for rocks with different types of stress dependent permeability is discussed in [15].

In this paper we present a numerical method for a fully coupled model that simulates single-phase flow through a deforming porous medium. The coupled equations used for the model were formulated on the basis of Darcy's law and the conservation principles for mass and linear momentum. We selected the isotropic, linear-elastic model to simulate the constitutive behaviour of reservoir rocks. The analysis of this type of model combining a mixed method and a continuous or discontinuous Galerkin method was considered e.g. in [6], [7], [11] and [12]. In the present article, the permeability tensor used in the model is stress-dependent. This introduces a nonlinear term and therein a novel analysis is required.

We will consider mixed finite elements (MFE) for the Darcy flow and Galerkin finite elements (CG) for elasticity. We present a priori optimal continuous in time and discrete in time error estimates.

Let $\Omega \subset \mathbb{R}^{d}, d=1,2$ or 3 , denote the domain of interest. The coupled balance equations are written as follow: find $(\mathbf{u}, p)$ such that

$$
\begin{aligned}
-(\lambda+\mu) \nabla(\nabla \cdot \mathbf{u})-\mu \nabla^{2} \mathbf{u}+\alpha \nabla p & =\mathbf{f} \text { in } \Omega \times(0, T] \\
\frac{\partial}{\partial t}\left(c_{0} p+\alpha \nabla \cdot \mathbf{u}\right)-\frac{1}{\mu_{f}} \nabla \cdot K\left(\sigma_{m}\right)\left(\nabla p-\rho_{f} \mathbf{g}\right) & =s_{f} \text { in } \Omega \times(0, T] \\
p & =p_{D} \text { on } \Gamma_{p} \times(0, T] \\
-\frac{1}{\mu_{f}} K\left(\sigma_{m}\right)\left(\nabla p-\rho_{f} \mathbf{g}\right) \cdot \eta & =\xi \text { on } \Gamma_{f} \times(0, T] \\
\mathbf{u} & =\mathbf{u}_{D} \text { on } \Gamma_{0} \times(0, T] \\
\tilde{\sigma} \eta & =\mathbf{r}_{N} \text { on } \Gamma_{N} \times(0, T] \\
p(0) & =p_{0} \text { in } \Omega,
\end{aligned}
$$

where $\partial \Omega=\Gamma_{p} \cup \Gamma_{f}$ and $\partial \Omega=\Gamma_{0} \cup \Gamma_{N}$, with meas $\left(\Gamma_{0}\right)>0$. The symbol $\eta$ represents the outward normal vector on $\partial \Omega$.

The physical parameters of the model are: $\lambda, \mu$, the Lamé constants, $c_{0}$, the constrained specific storage coefficient, $\alpha$, the Biot-Willis constant, $\mu_{f}$, 
the fluid viscosity, $\rho_{f}$, the fluid mass density and $\mathbf{g}$, the body force per unit of mass.

The primary variables are the pressure $p$ and the deformation $\mathbf{u}$. The effective stress $\sigma$, is the standard stress tensor from elasticity,

$$
\sigma(\mathbf{u})=2 \mu \epsilon(\mathbf{u})+\lambda \operatorname{tr}(\epsilon(\mathbf{u})) I,
$$

where

$$
\epsilon(\mathbf{u})=\frac{1}{2}\left(\operatorname{grad} \mathbf{u}+(\operatorname{grad} \mathbf{u})^{t}\right),
$$

and $I$ is the identity matrix in $\mathbb{R}^{d} \times \mathbb{R}^{d}, \sigma_{m}$ denotes the effective mean stress,

$$
\sigma_{m}=\frac{1}{d} \operatorname{tr}(\epsilon(\mathbf{u}))
$$

and the total stress, $\tilde{\sigma}$, is given by

$$
\tilde{\sigma}(\mathbf{u}, p)=\sigma(\mathbf{u})-\alpha p I .
$$

$K$ denotes the symmetric permeability tensor which is stress dependent. In [15], the stress dependent relationship between permeability and effective mean stress is defined for three common types of rocks, assuming the permeability tensor to be isotropic. The relationship between permeability and effective mean stress for the first type of rock is defined by $K=K_{0} e^{-k_{b} \sigma_{m}}$, where $k_{b}$ is a parameter characteristic of the rock that may be determined by experimental data, and $K_{0}$ is the reference permeability (i.e. the permeability of the rock sample measured under effective mean stress $\left.\sigma_{m}=0\right)$. For the second type of rock, the permeability is defined by $K=K_{0}\left(1-m \sigma_{m}\right)$, where $m$ is the variable that determines the reduction in permeability. For the third type of rock, the authors relate the permability with the rock porosity $\phi, K=K_{0}\left(\phi / \phi_{0}\right)^{n}$, where $K_{0}$ is the permability at reference porosity $\phi_{0}$ and $n$ is a variable that may be determined by experimental data. In the computational results presented in Section 5 we restrict our attention to rocks of the first type.

In practice, if the initial conditions $p_{0}$ is unknown, then $p_{0}$ can be found by considering $\nabla p(0)=\rho_{f} \mathbf{g}$ and then use the first equation of (1) to find $\mathbf{u}(0)$. This paper is organized as follows: in Section 2, we present the variational formulation of the problem. In Section 3 we describe and prove optimal error estimates for the numerical method in the continuous in time case. The fully discrete scheme and its error analysis is presented in Section 4. In Section 
5 we carry out numerical experiments. The last section concludes the paper with a summary and discussion of the main results.

\section{Variational formulation}

In this section we present the mixed variational formulation of the problem (1).

The space used for the flux variable is

$$
\mathbf{H}(\operatorname{div}):=\left\{\mathbf{s} \in\left(L^{2}(\Omega)\right)^{d}: \nabla \cdot \mathbf{s} \in L^{2}(\Omega)\right\}
$$

and we define its subset

$$
\mathbf{S}_{0}:=\left\{\mathbf{s} \in \mathbf{H}(\operatorname{div}):\left.\mathbf{s} \cdot \eta\right|_{\Gamma_{f}}=0\right\} .
$$

For the deformation we consider

$$
\mathbf{V}_{0}:=\left\{\mathbf{v} \in H^{1}((\Omega))^{d}:\left.\mathbf{v}\right|_{\Gamma_{0}}=0\right\}
$$

Associated to this space we define the bilinear form $a_{\mathbf{u}}(.,$.$) by$

$$
a_{\mathbf{u}}(\mathbf{u}, \mathbf{v}):=\int_{\Omega} \sigma(\mathbf{u}): \epsilon(\mathbf{v}) d \mathbf{x}
$$

or equivalently

$$
a_{\mathbf{u}}(\mathbf{u}, \mathbf{v})=\int_{\Omega}(2 \mu(\epsilon(\mathbf{u}): \epsilon(\mathbf{v}))+\lambda(\nabla \cdot \mathbf{u})(\nabla \cdot \mathbf{u})) d \mathbf{x} .
$$

The space $\mathbf{V}_{0}$ is endowed with the energy norm $\|\cdot\|_{a_{\mathbf{u}}}$ where $\|\mathbf{v}\|_{a_{\mathbf{u}}}^{2}:=a_{\mathbf{u}}(\mathbf{v}, \mathbf{v})$.

The bilinear form is continuous and coercive in $\mathbf{V}_{0} \times \mathbf{V}_{0}([2])$; therefore, for some positive real number $C_{\text {cont }}$ and $C_{\text {coer }}$ holds

$$
\begin{gathered}
a_{\mathbf{u}}(\mathbf{u}, \mathbf{v}) \leq C_{\text {cont }}\|\mathbf{u}\|_{H^{1}}\|\mathbf{v}\|_{H^{1}}, \forall \mathbf{u}, \mathbf{v} \in \mathbf{V}_{0}, \\
a_{\mathbf{u}}(\mathbf{v}, \mathbf{v}) \geq C_{\text {coer }}\|\mathbf{v}\|_{H^{1}}^{2}, \forall \mathbf{v} \in \mathbf{V}_{0} .
\end{gathered}
$$

In order to introduce the mixed formulation for the flow, we consider the variable for the flux $\mathbf{z}=-\frac{1}{\mu_{f}} K\left(\sigma_{m}\right)\left(\nabla p-\rho_{f} \mathbf{g}\right)$.

We define the linear functional

$$
\begin{aligned}
& \ell_{1}(\mathbf{v})=\int_{\Omega} \mathbf{f} \cdot \mathbf{v}+\int_{\Gamma_{N}} \mathbf{r}_{N} \cdot \mathbf{v}, \quad \mathbf{v} \in \mathbf{V}_{0}, \\
& \ell_{2}(w)=\int_{\Omega} s_{f} w, \quad w \in L^{2}(\Omega), \\
& \ell_{3}(\mathbf{s})=-\int_{\Gamma_{p}} p_{D} \mathbf{s} \cdot \eta+\int_{\Omega} \rho_{f} \mathbf{g} \cdot \mathbf{s}, \quad \mathbf{s} \in \mathbf{S}_{0} .
\end{aligned}
$$


Since the boundary conditions are allowed to be inhomogeneous, we need to select, for each $t \in[0, T]$, a function $\mathbf{u}_{d}(., t) \in\left(H^{1}(\Omega)\right)^{d}$ such that $\left.\mathbf{u}_{d}(., t)\right|_{\Gamma_{0}}=$ $\mathbf{u}_{D}(., t)$ and a function $\mathbf{z}_{d}(., t) \in \mathbf{H}(\operatorname{div})$ such that $\left.\mathbf{z}_{d}(., t)\right|_{\Gamma_{f}} \cdot \eta=\xi(., t)$.

The variational problem becomes: find $\mathbf{u} \in \mathbf{u}_{d}+H^{1}\left([0, T] ; \mathbf{V}_{0}\right), p \in$ $H^{1}\left([0, T] ; L^{2}(\Omega)\right)$ and $\mathbf{z} \in \mathbf{z}_{d}+L^{2}\left([0, T] ; \mathbf{S}_{0}\right)$ such that

$$
\begin{aligned}
a_{\mathbf{u}}(\mathbf{u}, \mathbf{v})-\alpha(\nabla \cdot \mathbf{v}, p) & =\ell_{1}(\mathbf{v}), \\
\left(c_{0} \frac{\partial p}{\partial t}, w\right)+\alpha\left(\frac{\partial}{\partial t} \nabla \cdot \mathbf{u}, w\right)+(\nabla \cdot \mathbf{z}, w) & =\ell_{2}(w), \\
\mu_{f}\left(K^{-1}\left(\sigma_{m}\right) \mathbf{z}, \mathbf{s}\right)-(p, \nabla \cdot \mathbf{s}) & =\ell_{3}(\mathbf{s})
\end{aligned}
$$

holds for all $(\mathbf{v}, w, \mathbf{s}) \in\left(\mathbf{V}_{0}, L^{2}(\Omega), \mathbf{S}_{0}\right)$ and $t \in[0, T]$.

We also make the following smoothness assumptions, in order the above variational formulation makes sense:

$$
\begin{aligned}
& \mathbf{f} \in C^{1}\left([0, T] ;\left(H^{-1}(\Omega)\right)^{d}\right), \\
& s_{f} \in C\left([0, T] ; L^{2}(\Omega)\right), \\
& p_{D} \in C\left([0, T] ; L^{2}\left(\Gamma_{p}\right)\right), \\
& \xi \in C([0, T] ; \operatorname{Tr} S), \quad \operatorname{Tr} S=\left\{\mathbf{s} \cdot \eta_{\left.\right|_{\Gamma_{f}}}: \mathbf{s} \in \mathbf{H}(\operatorname{div})\right\}, \\
& \mathbf{u}_{D} \in C^{1}\left([0, T] ;\left(H^{1 / 2}\left(\Gamma_{0}\right)\right)^{d}\right), \\
& \mathbf{r}_{N} \in C^{1}\left([0, T] ;\left(H^{-1 / 2}\left(\Gamma_{N}\right)^{d}\right)\right), \\
& \mathbf{g} \in C\left([0, T] ;\left(L^{2}(\Omega)\right)^{d}\right), \\
& \mathbf{u}_{0} \in\left(H^{1}(\Omega)\right)^{d} \\
& p_{0} \in L^{2}(\Omega) .
\end{aligned}
$$

For the permeability tensor we require that

$$
K^{-1}\left(\sigma_{m}\right) \in C\left([0, T] ;\left(L^{\infty}(\Omega)\right)^{d \times d}\right),
$$

and for simplicity we assume the storage coefficient to be strictly positive and uniformly bounded:

$$
0<\gamma_{c} \leq c_{o}(\mathbf{x}) \leq L_{c}, \forall \mathbf{x} \in \Omega .
$$

A more refined analysis in required to treat $c_{o}(\mathbf{x}) \geq 0$ (see [10]).

\section{Semi-discrete approximation}

In this section we define the semi-discrete approximation, continuous in time, for our initial boundary problem. In what follows, $C$ will be a generic positive constant with different values. In order to approximate the variational problem (2)-(4) with a finite element scheme we need to provide some definitions. 
Let $\mathcal{E}_{h}$ and $\mathcal{E}_{H}$ be two nondegenerate partitions of the polyhedral domain $\Omega$, with maximal element diameter $h$ and $H$, respectively. The elements of $\mathcal{E}_{h}$ and $\mathcal{E}_{H}$ are triangles or convex quadrilaterals, if $d=2$, and tetrahedra, hexahedra or prisms, if $d=3$. Let $\left(W_{h}, \mathbf{S}_{h}\right) \subset\left(L^{2}(\Omega) \times \mathbf{H}(\operatorname{div})\right)$ denote a standard mixed finite element space (see e.g. [3]) on $\mathcal{E}_{h}$, of order $k$, and

$$
\mathbf{S}_{h, 0}:=\left\{\mathbf{s} \in \mathbf{S}_{h}: \mathbf{s} \cdot \eta_{\left.\right|_{\Gamma_{f}}}=0\right\} .
$$

We consider linear operators $\Pi_{h}: \mathbf{H}(\operatorname{div}) \rightarrow \mathbf{S}_{h}$ and $P_{h}: L^{2} \rightarrow W_{h}$ which satisfy the following properties:

$$
\begin{aligned}
\left(\nabla \cdot\left(\mathbf{s}-\Pi_{h} \mathbf{s}\right), w\right) & =0, \quad \forall w \in W_{h}, \\
\left\|\mathbf{s}-\Pi_{h} \mathbf{s}\right\|_{L^{2}(\Omega)} & \leq C h^{\kappa}\|\mathbf{s}\|_{H^{\kappa}(\Omega)}, \quad 1 \leq \kappa \leq k+1, \\
\nabla \cdot \Pi_{h} & =P_{h} \nabla \cdot, \\
\left(\nabla \cdot \mathbf{s}_{h}, p-P_{h} p\right) & =0, \quad \forall \mathbf{s}_{h} \in \mathbf{S}_{h}, \\
\left\|p-P_{h} p\right\|_{L^{2}(\Omega)} & \leq C h^{\kappa}\|p\|_{H^{\kappa}(\Omega)}, \quad 0 \leq \kappa \leq k+1 .
\end{aligned}
$$

The above conditions are crucial for deriving our results. Examples of mixed spaces with these properties are the Raviart-Thomas-Nedelec (RTN) spaces $([9],[16])$.

Let $\mathbf{V}_{H}$ be the space of continuous piecewise polynomials of degree $r$ defined on $\mathcal{E}_{H}$ and

$$
\mathbf{V}_{H, 0}:=\left\{\mathbf{v} \in \mathbf{V}_{H}:\left.\mathbf{v}\right|_{\Gamma_{0}}=0\right\} .
$$

The elliptic projector $\tilde{P}:\left(H^{1}(\Omega)\right)^{d} \rightarrow \mathbf{V}_{H}$ is defined by

$$
a_{\mathbf{u}}\left(\mathbf{u}-\tilde{P} \mathbf{u}, \mathbf{v}_{H}\right)=0, \forall \mathbf{v}_{H} \in \mathbf{V}_{H}
$$

and satisfies (see [2])

$$
\|\mathbf{u}-\tilde{P} \mathbf{u}\|_{a_{\mathbf{u}}} \leq C H^{\tau}\|\mathbf{u}\|_{\left(H^{\tau+1}(\Omega)\right)^{d}}, \quad 0 \leq \tau \leq r .
$$

For each $t \in[0, T]$, we define the cut-off operator $\mathcal{M}$ as

$$
\mathcal{M}(\rho)(\mathbf{x}, t)=\min (|\rho(\mathbf{x}, t)|, M) \text { a.e. in } \Omega,
$$

where the constant $M$ is sufficiently large in order to satisfy

$$
\mathcal{M}\left(\sigma_{m}\right)(\mathbf{x}, t)=\sigma_{m}(\mathbf{x}, t), \text { a.e. in } \Omega .
$$

The cut-off operator $\mathcal{M}$ is introduced in the numerical scheme in order to derive convergence estimates. More precisely, we use the properties (15) and (14) below in the convergence proof. Those two conditions motivated the 
definition of $\mathcal{M}$. A similar operator is used in [14] for a coupled flow and reactive transport problem.

It is straightforward to prove that the cut-off operator $\mathcal{M}$ is uniformly Lipschitz continuous:

$$
\|\mathcal{M}(\rho)-\mathcal{M}(\varsigma)\|_{L^{2}(\Omega)} \leq\|\rho-\varsigma\|_{L^{2}(\Omega)}, \forall \rho, \varsigma \in L^{2}(\Omega) .
$$

The inequalities (12) and (15) can easily to be shown to be valid for rocks of types one and two. We require the existence of the inverse of the operator $K$ and we assume that $K^{-1}(\mathcal{M}(\rho))$ is positive definite for all $\rho \in L^{2}(\Omega)$; that is, there exists a positive constant $\zeta$ such that, for all $\mathbf{s} \in\left(L^{2}(\Omega)\right)^{d}$,

$$
\left(K^{-1}(\mathcal{M}(\rho)) \mathbf{s}, \mathbf{s}\right) \geq \zeta\|\mathbf{s}\|_{L^{2}(\Omega)} .
$$

We also make the assumption that $K^{-1}$ is a uniformly Lipschitz continuous function with Lipschitz constant $L$, and using (13), we see that

$$
\left\|K^{-1}(\mathcal{M}(\rho))-K^{-1}(\mathcal{M}(\xi))\right\| \leq L\|\rho-\varsigma\|_{L^{2}(\Omega)}, \forall \rho, \varsigma \in L^{2}(\Omega) .
$$

Let $\overline{\mathbf{u}}_{d}(\mathbf{x}, t)=\tilde{P} \mathbf{u}_{d}(\mathbf{x}, t)$ and $\overline{\mathbf{z}}_{d}(\mathbf{x}, t)=\Pi_{h} \mathbf{z}_{d}(\mathbf{x}, t)$.

The finite element method becomes: find $\overline{\mathbf{u}} \in \overline{\mathbf{u}}_{d}+H^{1}\left([0, T] ; \mathbf{V}_{H, 0}\right), \bar{p} \in$ $H^{1}\left([0, T] ; W_{h}\right)$ and $\overline{\mathbf{z}} \in \overline{\mathbf{z}}_{d}+L^{2}\left([0, T] ; \mathbf{S}_{h, 0}\right)$

$$
\begin{aligned}
a_{\mathbf{u}}(\overline{\mathbf{u}}, \mathbf{v})-\alpha(\bar{p}, \nabla \cdot \mathbf{v}) & =\ell_{1}(\mathbf{v}), \\
\left(c_{0} \bar{p}_{t}, w\right)+\alpha\left(\nabla \cdot \overline{\mathbf{u}}_{t}, w\right)+(\nabla \cdot \overline{\mathbf{z}}, w) & =\ell_{2}(w), \\
\mu_{f}\left(K^{-1}\left(\mathcal{M}\left(\bar{\sigma}_{m}\right)\right) \overline{\mathbf{z}}, \mathbf{s}\right)-(\bar{p}, \nabla \cdot \mathbf{s}) & =\ell_{3}(\mathbf{s}),
\end{aligned}
$$

holds for every $t \in[0, T]$ and $(\mathbf{v}, w, \mathbf{s}) \in\left(\mathbf{V}_{H, 0}, W_{h}, \mathbf{S}_{h, 0}\right)$. Here $\bar{\sigma}_{m}$ is defined locally in $\mathcal{E}_{H}$ by

$$
\bar{\sigma}_{m}=\frac{1}{d} \operatorname{tr}(\epsilon(\overline{\mathbf{u}})) .
$$

Additionally, we consider the initial conditions

$$
\begin{gathered}
a_{\mathbf{u}}(\overline{\mathbf{u}}, \mathbf{v})_{\left.\right|_{t=0}}=a_{\mathbf{u}}\left(\mathbf{u}_{0}, \mathbf{v}\right), \forall \mathbf{v}_{H} \in \mathbf{V}_{H}, \\
(\bar{p}, w)_{\left.\right|_{t=0}}=\left(p_{0}, w\right), \forall w \in W_{h} .
\end{gathered}
$$


3.1. Existence and uniqueness. Results of existence, uniqueness and regularity for the linear continuous problem can be found in [13]. We now establish the existence and uniqueness of a solution of semi-discrete problem (16)-(18). For the proof, we essentially follow [11]. However a special attention needs to be given due to treatment of the nonlinearity

By construction, we can represent any function in $\mathbf{V}_{H}, W_{h}$ or $\mathbf{S}_{h}$ as a linear combination of the respective basis functions.

Let $\mathbf{V}_{H}=<\mathbf{v}_{1}, \mathbf{v}_{2}, \ldots, \mathbf{v}_{n_{\mathbf{u}}}>, W_{h}=<w_{1}, w_{2}, \ldots, w_{n_{p}}>$ and $\mathbf{S}_{h}=<$ $\mathbf{s}_{1}, \mathbf{s}_{2}, \ldots, \mathbf{s}_{n_{\mathbf{z}}}>$. We write the functions $\overline{\mathbf{u}}, \bar{p}$ and $\overline{\mathbf{z}}$ as components of their respective basis functions:

$$
\begin{aligned}
\overline{\mathbf{u}}(\mathbf{x}, t) & =\sum_{j=1}^{n_{\mathbf{u}}} \mathbf{u}_{j}(t) \mathbf{v}_{j}(\mathbf{x})+\sum_{j=1}^{n_{\mathbf{u}}} \mathbf{u}_{d, j}(t) \mathbf{v}_{j}(\mathbf{x}), \\
\bar{p}(\mathbf{x}, t) & =\sum_{j=1}^{n_{p}} p_{j}(t) w_{j}(\mathbf{x}), \\
\overline{\mathbf{z}}(\mathbf{x}, t) & =\sum_{j=1}^{n_{\mathbf{z}}} \mathbf{z}_{j}(t) \mathbf{s}_{j}(\mathbf{x})+\sum_{j=1}^{n_{\mathbf{z}}} \mathbf{z}_{d, j}(t) \mathbf{s}_{j}(\mathbf{x}) .
\end{aligned}
$$

The vector $\mathbf{u}_{d H}(t)=\left[\mathbf{u}_{d, 1}(t), \ldots, \mathbf{u}_{d, n_{\mathbf{u}}}(t)\right]^{T}$ has the components of the function $\overline{\mathbf{u}}_{d}$ and $\mathbf{z}_{d h}(t)=\left[\mathbf{z}_{d, 1}(t), \ldots, \mathbf{z}_{d, n_{\mathbf{z}}}(t)\right]^{T}$ has the components of $\overline{\mathbf{z}}_{d}$, which come from the given boundary conditions. Let $\mathbf{u}_{H}(t)=\left[\mathbf{u}_{1}(t), \ldots, \mathbf{u}_{n_{\mathbf{u}}}(t)\right]^{T}$, $\mathbf{p}_{h}(t)=\left[p_{1}(t), \ldots, p_{n_{p}}(t)\right]^{T}$ and $\mathbf{z}_{h}(t)=\left[\mathbf{z}_{1}(t), \ldots, \mathbf{z}_{n_{\mathbf{z}}}(t)\right]^{T}$.

Substituting (19)-(21) in (16)-(18) we observe that we rewrite the problem in a matrix form: find $\mathbf{u}_{H}(t), \mathbf{p}_{h}(t)$ and $\mathbf{z}_{h}(t)$ such that

$$
\begin{aligned}
A_{\mathbf{u u}} \mathbf{u}_{H}-\alpha A_{p \mathbf{u}} \mathbf{p}_{h} & =\mathbf{l}_{1} \\
c_{0} A_{p p} \frac{\partial \mathbf{p}_{h}}{\partial t}+\alpha A_{p \mathbf{u}}^{T} \frac{\partial \mathbf{u}_{H}}{\partial t}+A_{\mathbf{z p}} \mathbf{z}_{h} & =\mathbf{l}_{2} \\
A_{\mathbf{z z}}\left(\bar{\sigma}_{m}\right) \mathbf{z}_{h}-A_{p \mathbf{z}} \mathbf{p}_{h} & =\mathbf{l}_{3} .
\end{aligned}
$$

Since $A_{\mathbf{u u}}$ is nonsingular, equation (22) is equivalent to

$$
\mathbf{u}_{H}=A_{\mathbf{u u}}^{-1} \mathbf{l}_{1}+\alpha A_{\mathbf{u u}}^{-1} A_{p \mathbf{u}} \mathbf{p}_{h} .
$$

Differentiating with respect to time we obtain

$$
\frac{\partial \mathbf{u}_{H}}{\partial t}=A_{\mathbf{u u}}^{-1} \frac{\partial \mathbf{l}_{1}}{\partial t}+\alpha A_{\mathbf{u u}}^{-1} A_{p \mathbf{u}} \frac{\partial \mathbf{p}_{h}}{\partial t} .
$$


Condition (14) ensures the nonsingularity of $A_{\mathbf{z z}}\left(\bar{\sigma}_{m}\right)$ and then the equation (22) can be rewritten in the form

$$
\mathbf{z}_{h}=\left(A_{\mathbf{z z}}\left(\bar{\sigma}_{m}\right)\right)^{-1} \mathbf{l}_{3}+A_{\mathbf{z z}}^{-1}\left(\bar{\sigma}_{m}\right) A_{\mathbf{z} p}^{T} \mathbf{p}_{h} .
$$

Using (25), we can write $\bar{\sigma}_{m}$ as a function of $\mathbf{p}_{h}, \bar{\sigma}_{m}=F\left(\mathbf{p}_{h}\right)$.

From (23) we obtain

$$
\begin{aligned}
& \left(c_{0} A_{p p}+\alpha A_{p \mathbf{u}}^{T} A_{\mathbf{u u}}^{-1} A_{p \mathbf{u}}\right) \frac{\partial \mathbf{p}_{h}}{\partial t} \\
& =\mathbf{l}_{2}-\alpha A_{p \mathbf{u}}^{T} A_{\mathbf{u u}}^{-1} \frac{\partial \mathbf{l}_{1}}{\partial t}-A_{\mathbf{z} p}\left(A_{\mathbf{z z}}\left(F\left(\mathbf{p}_{h}\right)\right)\right)^{-1} \mathbf{l}_{3}-A_{\mathbf{z} p}\left(A_{\mathbf{z z}}\left(F\left(\mathbf{p}_{h}\right)\right)\right)^{-1} A_{\mathbf{z} p}^{T} \mathbf{p}_{h} .
\end{aligned}
$$

The matrix multiplying $\frac{\partial \mathbf{p}_{h}}{\partial t}$ is symmetric and positive definite and the right hand side of the resulting equation is continuous in time. Therefore, together with the given initial condition for $p$ and $\mathbf{u}$, we obtain an initial value problem. Thus, by Peano's existence theorem for ordinary differential equations, there exists at least one solution of the equation on $[0, \epsilon]$, for some $\epsilon>0$.

For the proof of the uniqueness of the solution of the problem (16)-(18), we follow [11] and use the property (14).

3.2. Continuous in time a priori error estimate. In this section we examine the a priori error estimates for the semi-discretization previously defined. Even if this approximation is not computed in practice, this analysis provides information about the quality of the spatial discretization. The fully discrete approximation will be presented in the next section.

The analysis we employ is an extension of the techniques presented in [11] to the case of stress dependent permeability.

For the study of the error, we assume that the weak solution of the problem (2)-(4) is sufficiently regular, e.g. we assume that $\mathbf{u} \in L^{\infty}\left([0, T] ;\left(H^{r+1}(\Omega)\right)^{d}\right)$, $\mathbf{u}_{t} \in L^{2}\left([0, T] ;\left(H^{r+1}(\Omega)\right)^{d}\right), p \in L^{\infty}\left([0, T] ; L^{2}(\Omega)\right) \cap L^{2}\left([0, T] ; H^{k+1}(\Omega)\right), p_{t}$ $\in L^{2}\left([0, T] ; H^{k+1}(\Omega)\right)$ and $\mathbf{z} \in L^{2}\left([0, T] ;\left(H^{k+1}(\Omega)\right)^{d}\right)$. The order of the error in the convergent results, which we will establish in this section, depends on $r$ and $k$.

To simplify the notation in what follows we use $\|\cdot\|_{0},\|\cdot\|_{\infty}$ and $\|.\|_{1}$, respectively, for the $L^{2}, L^{\infty}$ and $H^{1}$ norms.

In the next theorem we present auxiliary error estimates. With this result, the problem of finding error bounds will be reduced to applying the interpolation theory in Hilbert spaces, presented in the beginning of this section. 
Theorem 1. Let $(\mathbf{u}, p, \mathbf{z})$ be the solution of (2)-(4) and $(\overline{\mathbf{u}}, \bar{p}, \overline{\mathbf{z}})$ be the solution of (16)-(18). Then

$$
\|\tilde{P} \mathbf{u}-\overline{\mathbf{u}}\|_{L^{\infty}\left(H^{1}\right)}^{2}+\left\|P_{h} p-\bar{p}\right\|_{L^{\infty}\left(L^{2}\right)}^{2}+\left\|\Pi_{h} \mathbf{z}-\overline{\mathbf{z}}\right\|_{L^{2}\left(L^{2}\right)}^{2} \leq C\left(H^{2 r}+h^{2 k+2}\right),
$$

where $C$ depends on the model parameters, $T, \zeta, L, \gamma_{c}, C_{\text {coer }}, p, p_{t}, \mathbf{u}, \mathbf{u}_{t}$ and on $M$ being sufficiently large but is not dependent on $H$ and $h$.

Proof For the simplicity in the analysis, we assume that $\mathbf{u}_{0}, \mathbf{u}_{d} \in \mathbf{V}_{H}, p_{0} \in$ $W_{h}$ and $\mathbf{z}_{d} \in \mathbf{S}_{h}$. This assumption does not affect the rate of convergence. The following equations

$$
\begin{aligned}
a_{\mathbf{u}}(\mathbf{u}-\overline{\mathbf{u}}, \mathbf{v})-\alpha(p-\bar{p}, \nabla \cdot \mathbf{v}) & =0 \\
\left(c_{0}(p-\bar{p})_{t}, w\right)+\alpha\left(\nabla \cdot(\mathbf{u}-\overline{\mathbf{u}})_{t}, w\right)+(\nabla \cdot(\mathbf{z}-\overline{\mathbf{z}}), w) & =0 \\
\mu_{f}\left(K^{-1}\left(\sigma_{m}\right) \mathbf{z}-K^{-1}\left(\mathcal{M}\left(\bar{\sigma}_{m}\right)\right) \overline{\mathbf{z}}, \mathbf{s}\right)-(p-\bar{p}, \nabla \cdot \mathbf{s}) & =0
\end{aligned}
$$

hold for all $(\mathbf{v}, w, \mathbf{s}) \in\left(\mathbf{V}_{H, 0}, W_{h}, \mathbf{S}_{h, 0}\right)$.

Let $\mathbf{v}=(\tilde{P} \mathbf{u}-\overline{\mathbf{u}})_{t}, w=P_{h} p-\bar{p}$ and $\mathbf{s}=\Pi_{h} \mathbf{z}-\overline{\mathbf{z}}$. Using in (27) the chain rule in time, the symmetry of $a_{\mathbf{u}}$ and noticing that $a_{\mathbf{u}}\left(\mathbf{u}-\tilde{P} \mathbf{u},(\tilde{P} \mathbf{u}-\overline{\mathbf{u}})_{t}\right)=0$, we obtain

$$
\begin{aligned}
& \frac{1}{2} \frac{\partial}{\partial t} a_{\mathbf{u}}(\tilde{P} \mathbf{u}-\overline{\mathbf{u}}, \tilde{P} \mathbf{u}-\overline{\mathbf{u}})-\alpha\left(p-P_{h} p, \nabla \cdot(\tilde{P} \mathbf{u}-\overline{\mathbf{u}})_{t}\right) \\
&-\alpha\left(P_{h} p-\bar{p}, \nabla \cdot(\tilde{P} \mathbf{u}-\overline{\mathbf{u}})_{t}\right)=0 .
\end{aligned}
$$

From (28), (29), and using the fact that

$$
\left(\nabla \cdot\left(\mathbf{z}-\Pi_{h} \mathbf{z}\right), P_{h} p-\bar{p}\right)=0 \text { and }\left(p-P_{h} p, \nabla \cdot\left(\Pi_{h} \mathbf{z}-\overline{\mathbf{z}}\right)\right)=0,
$$

we obtain, respectively,

$$
\begin{aligned}
&\left(c_{0}\left(p-P_{h} p\right)_{t}, P_{h} p-\bar{p}\right)+\left(c_{0}\left(P_{h} p-\bar{p}\right)_{t}, P_{h} p-\bar{p}\right) \\
&+\alpha\left(\nabla \cdot(\mathbf{u}-\tilde{P} \mathbf{u})_{t}, P_{h} p-\bar{p}\right)+ \alpha\left(\nabla \cdot(\tilde{P} \mathbf{u}-\overline{\mathbf{u}})_{t}, P_{h} p-\bar{p}\right) \\
&+\left(\nabla \cdot\left(\Pi_{h} \mathbf{z}-\overline{\mathbf{z}}\right), P_{h} p-\bar{p}\right)=0
\end{aligned}
$$

and

$$
\mu_{f}\left(K^{-1}\left(\sigma_{m}\right) \mathbf{z}-K^{-1}\left(\mathcal{M}\left(\bar{\sigma}_{m}\right)\right) \overline{\mathbf{z}}, \Pi_{h} \mathbf{z}-\overline{\mathbf{z}}\right)-\left(P_{h} p-\bar{p}, \nabla \cdot\left(\Pi_{h} \mathbf{z}-\overline{\mathbf{z}}\right)\right)=0 .
$$


Summing (31) and (32) and again applying the chain rule, we obtain

$$
\begin{array}{r} 
\\
\frac{1}{2} \frac{\partial}{\partial t}\left(c_{0}\left(P_{h} p-\bar{p}\right), P_{h} p-\bar{p}\right)+\alpha\left(\nabla \cdot(\tilde{P} \mathbf{u}-\overline{\mathbf{u}})_{t}, P_{h} p-\bar{p}\right) \\
+\mu_{f}\left(K^{-1}\left(\sigma_{m}\right) \mathbf{z}-K^{-1}\left(\mathcal{M}\left(\bar{\sigma}_{m}\right)\right) \overline{\mathbf{z}}, \Pi_{h} \mathbf{z}-\overline{\mathbf{z}}\right) \\
=-\left(c_{0}\left(p-P_{h} p\right)_{t}, P_{h} p-\bar{p}\right)-\alpha\left(\nabla \cdot(\mathbf{u}-\tilde{P} \mathbf{u})_{t}, P_{h} p-\bar{p}\right) .
\end{array}
$$

Summing (30) and (33), yields

$$
\begin{array}{r}
\frac{1}{2} \frac{\partial}{\partial t} a_{\mathbf{u}}(\tilde{P} \mathbf{u}-\overline{\mathbf{u}}, \tilde{P} \mathbf{u}-\overline{\mathbf{u}})+\frac{1}{2} \frac{\partial}{\partial t}\left(c_{0}\left(P_{h} p-\bar{p}\right), P_{h} p-\bar{p}\right) \\
+\mu_{f}\left(K^{-1}\left(\sigma_{m}\right) \mathbf{z}-K^{-1}\left(\mathcal{M}\left(\bar{\sigma}_{m}\right)\right) \overline{\mathbf{z}}, \Pi_{h} \mathbf{z}-\overline{\mathbf{z}}\right) \\
=\alpha\left(\nabla \cdot(\tilde{P} \mathbf{u}-\overline{\mathbf{u}})_{t}, p-P_{h} p\right)-\left(c_{0}\left(p-P_{h} p\right)_{t}, P_{h} p-\bar{p}\right) \\
-\alpha\left(\nabla \cdot(\mathbf{u}-\tilde{P} \mathbf{u})_{t}, P_{h} p-\bar{p}\right) .
\end{array}
$$

In (34) we use the following equality

$$
\begin{array}{r}
K^{-1}\left(\sigma_{m}\right) \mathbf{z}-K^{-1}\left(\mathcal{M}\left(\bar{\sigma}_{m}\right)\right) \overline{\mathbf{z}}=K^{-1}\left(\sigma_{m}\right)\left(\mathbf{z}-\Pi_{h} \mathbf{z}\right) \\
+\left(K^{-1}\left(\sigma_{m}\right)-K^{-1}\left(\mathcal{M}\left(\bar{\sigma}_{m}\right)\right)\right) \Pi_{h} \mathbf{z}+K^{-1}\left(\mathcal{M}\left(\bar{\sigma}_{m}\right)\right)\left(\Pi_{h} \mathbf{z}-\overline{\mathbf{z}}\right),
\end{array}
$$

and then, summing (33) and (34), integrating from 0 to $T$ and using the assumptions $(\tilde{P} \mathbf{u}-\overline{\mathbf{u}})(0)=0$ and $\left(P_{h} p-\bar{p}\right)(0)=0$, we deduce the result

$$
\begin{array}{r}
\frac{1}{2}\left[a_{\mathbf{u}}(\tilde{P} \mathbf{u}-\overline{\mathbf{u}}, \tilde{P} \mathbf{u}-\overline{\mathbf{u}})+\left(c_{0}\left(P_{h} p-\bar{p}\right), P_{h} p-\bar{p}\right)\right]_{\mid t=T} \\
+\int_{0}^{T} \mu_{f}\left(K^{-1}\left(\mathcal{M}\left(\bar{\sigma}_{m}\right)\right)\left(\Pi_{h} \mathbf{z}-\overline{\mathbf{z}}\right), \Pi_{h} \mathbf{z}-\overline{\mathbf{z}}\right)(\tau) d \tau \\
=\Phi_{1}+\Phi_{2}+\Phi_{3}+\Phi_{4}
\end{array}
$$

where

$$
\begin{gathered}
\Phi_{1}=-\int_{0}^{T} \mu_{f}\left(\left(K^{-1}\left(\sigma_{m}\right)-K^{-1}\left(\mathcal{M}\left(\bar{\sigma}_{m}\right)\right)\right) \Pi_{h} \mathbf{z}, \Pi_{h} \mathbf{z}-\overline{\mathbf{z}}\right)(\tau) d \tau \\
-\int_{0}^{T} \mu_{f}\left(K^{-1}\left(\sigma_{m}\right)\left(\mathbf{z}-\Pi_{h} \mathbf{z}\right), \Pi_{h} \mathbf{z}-\overline{\mathbf{z}}\right)(\tau) d \tau \\
\Phi_{2}=-\int_{0}^{T} \alpha\left(\nabla \cdot(\mathbf{u}-\tilde{P} \mathbf{u})_{t}, P_{h} p-\bar{p}\right)(\tau) d \tau \\
\Phi_{3}=\int_{0}^{T} \alpha\left(\nabla \cdot(\tilde{P} \mathbf{u}-\overline{\mathbf{u}})_{t}, p-P_{h} p\right)(\tau) d \tau,
\end{gathered}
$$




$$
\Phi_{4}=-\int_{0}^{T}\left(c_{0}\left(p-P_{h} p\right)_{t}, P_{h} p-\bar{p}\right)(\tau) d \tau
$$

Cauchy-Schwartz and Young's inequalities are now applied in bounding the above inequalities.

Using (12), (15) and the Lipschitz continuity of $K^{-1}$ we obtain

$$
\begin{aligned}
\Phi_{1} \leq & \frac{L}{2 \epsilon_{1}} \int_{0}^{T}\left\|\left(\sigma_{m}-\bar{\sigma}_{m}\right)(\tau)\right\|_{0}^{2}\left\|\Pi_{h} \mathbf{z}(\tau)\right\|_{\infty}^{2} d \tau+\frac{\epsilon_{1}}{2} \int_{0}^{T}\left\|\left(\Pi_{h} \mathbf{z}-\overline{\mathbf{z}}\right)(\tau)\right\|_{0}^{2} d \tau \\
& +\frac{\mu_{f}^{2}}{2 \epsilon_{1}} \int_{0}^{T}\left\|K^{-1}\left(\sigma_{m}\right)\right\|_{\infty}^{2}\left\|\left(\mathbf{z}-\Pi_{h} \mathbf{z}\right)(\tau)\right\|_{0}^{2} d \tau+\frac{\epsilon_{1}}{2} \int_{0}^{T}\left\|\left(\Pi_{h} \mathbf{z}-\overline{\mathbf{z}}\right)(\tau)\right\|_{0}^{2} d \tau \\
\leq & \frac{L}{2 d \epsilon_{1}}(2 \mu+3 \lambda)^{2} \int_{0}^{T}\|(\mathbf{u}-\overline{\mathbf{u}})(\tau)\|_{1}^{2}\left\|\Pi_{h} \mathbf{z}(\tau)\right\|_{\infty}^{2} d \tau \\
& +\frac{\mu_{f}^{2}}{2 \epsilon_{1}} \int_{0}^{T}\left\|K^{-1}\left(\sigma_{m}\right)\right\|_{\infty}^{2}\left\|\left(\mathbf{z}-\Pi_{h} \mathbf{z}\right)(\tau)\right\|_{0}^{2}+\epsilon_{1} \int_{0}^{T}\left\|\left(\Pi_{h} \mathbf{z}-\overline{\mathbf{z}}\right)(\tau)\right\|_{0}^{2} d \tau \\
\leq & \frac{L}{2 d \epsilon_{1}}(2 \mu+3 \lambda)^{2} \int_{0}^{T}\|(\mathbf{u}-\tilde{P} \mathbf{u})(\tau)\|_{1}^{2}\left\|\Pi_{h} \mathbf{z}(\tau)\right\|_{\infty}^{2} d \tau \\
& +\frac{L}{2 d \epsilon_{1}}(2 \mu+3 \lambda)^{2} \int_{0}^{T}\|(\tilde{P} \mathbf{u}-\overline{\mathbf{u}})(\tau)\|_{1}^{2}\left\|\Pi_{h} \mathbf{z}(\tau)\right\|_{\infty}^{2} d \tau \\
& +\frac{\mu_{f}^{2}}{2 \epsilon_{1}} \int_{0}^{T}\left\|K^{-1}\left(\sigma_{m}\right)\right\|_{\infty}^{2}\left\|\left(\mathbf{z}-\Pi_{h} \mathbf{z}\right)(\tau)\right\|_{0}^{2}+\epsilon_{1} \int_{0}^{T}\left\|\left(\Pi_{h} \mathbf{z}-\overline{\mathbf{z}}\right)(\tau)\right\|_{0}^{2} d \tau
\end{aligned}
$$

where $\epsilon_{1}$ is an arbitrary small constant.

For $\Phi_{2}$ we obtain

$$
\begin{aligned}
\Phi_{2} & \leq \alpha \int_{0}^{T}\left\|\nabla \cdot(\mathbf{u}-\tilde{P} \mathbf{u})_{t}(\tau)\right\|_{0}\left\|\left(P_{h} p-\bar{p}\right)(\tau)\right\|_{0} d \tau \\
& \leq \frac{\alpha^{2}}{2} \int_{0}^{T}\left\|\nabla \cdot(\mathbf{u}-\tilde{P} \mathbf{u})_{t}(\tau)\right\|_{0}^{2} d \tau+\frac{1}{2} \int_{0}^{T}\left\|\left(P_{h} p-\bar{p}\right)(\tau)\right\|_{0}^{2} d \tau
\end{aligned}
$$


To bound $\Phi_{3}$, we integrate by parts in time and observe that

$$
\begin{aligned}
\Phi_{3}= & -\int_{0}^{T} \alpha\left(\nabla \cdot(\tilde{P} \mathbf{u}-\overline{\mathbf{u}}),\left(p-P_{h} p\right)_{t}\right)(\tau) d \tau \\
& +\alpha\left(\nabla \cdot(\tilde{P} \mathbf{u}-\overline{\mathbf{u}}),\left(p-P_{h} p\right)\right)_{\left.\right|_{t=T}} \\
\leq & \int_{0}^{T} \alpha\|\nabla \cdot(\tilde{P} \mathbf{u}-\overline{\mathbf{u}})(\tau)\|_{0}\left\|\left(p-P_{h} p\right)_{t}(\tau)\right\|_{0} d \tau \\
& +\alpha\|\nabla \cdot(\tilde{P} \mathbf{u}-\overline{\mathbf{u}})(T)\|_{0}\left\|\left(p-P_{h} p\right)(T)\right\|_{0} \\
\leq & \frac{\alpha^{2}}{2} \int_{0}^{T}\|(\tilde{P} \mathbf{u}-\overline{\mathbf{u}})(\tau)\|_{1}^{2} d \tau+\frac{1}{2} \int_{0}^{T}\left\|\left(p-P_{h} p\right)_{t}(\tau)\right\|_{0}^{2} d \tau \\
& +\epsilon_{2}\|(\tilde{P} \mathbf{u}-\overline{\mathbf{u}})(T)\|_{1}^{2}+\frac{\alpha^{2}}{4 \epsilon_{2}}\left\|\left(p-P_{h} p\right)(T)\right\|_{0}^{2},
\end{aligned}
$$

where $\epsilon_{2}$ is an arbitrary small constant.

For $\Phi_{4}$ we obtain

$$
\begin{aligned}
\Phi_{4} & =-\int_{0}^{T}\left(c_{0}^{1 / 2}\left(p-P_{h} p\right)_{t}, c_{0}^{1 / 2}\left(P_{h} p-\bar{p}\right)\right)(\tau) d \tau \\
& \leq \frac{1}{2} \int_{0}^{T}\left\|c_{0}^{1 / 2}\left(p-P_{h} p\right)_{t}(\tau)\right\|_{0}^{2} d \tau+\frac{1}{2} \int_{0}^{T}\left\|c_{0}^{1 / 2}\left(P_{h} p-\bar{p}\right)(\tau)\right\|_{0}^{2} d \tau
\end{aligned}
$$

From (35), using the above estimates, the coercivity of $a_{\mathbf{u}}$ :

$$
a_{\mathbf{u}}(\tilde{P} \mathbf{u}-\overline{\mathbf{u}}, \tilde{P} \mathbf{u}-\overline{\mathbf{u}})_{\left.\right|_{t=T}} \geq C_{\text {coer }}\|(\tilde{P} \mathbf{u}-\overline{\mathbf{u}})(T)\|_{1}^{2}
$$


and the assumptions (5), (6) and (14), we find the inequality

$$
\begin{aligned}
& \left(\frac{1}{2} C_{\text {coer }}-\epsilon_{2}\right)\|(\tilde{P} \mathbf{u}-\overline{\mathbf{u}})(T)\|_{1}^{2}+\frac{\gamma_{c}}{2}\left\|\left(P_{h} p-\bar{p}\right)(T)\right\|_{0}^{2} \\
& +\left(\mu_{f} \zeta-\epsilon_{1}\right) \int_{0}^{T}\left\|\left(\Pi_{h} \mathbf{z}-\overline{\mathbf{z}}\right)(\tau)\right\|_{0}^{2} d \tau \\
\leq & \frac{L(2 \mu+3 \lambda)^{2}}{2 d \epsilon_{1}}\left(\int_{0}^{T}\|(\mathbf{u}-\tilde{P} \mathbf{u})(\tau)\|_{1}^{2} d \tau+\int_{0}^{T}\|(\tilde{P} \mathbf{u}-\overline{\mathbf{u}})(\tau)\|_{1}^{2} d \tau\right) \\
& +\frac{\mu_{f}^{2}}{2 \epsilon_{1}} \int_{0}^{T}\left\|K^{-1}\left(\sigma_{m}\right)\right\|_{\infty}^{2}\left\|\left(\mathbf{z}-\Pi_{h} \mathbf{z}\right)(\tau)\right\|_{0}^{2}+\frac{\alpha^{2}}{2} \int_{0}^{T}\left\|(\mathbf{u}-\tilde{P} \mathbf{u})_{t}(\tau)\right\|_{1}^{2} d \tau \\
& +\frac{1}{2} \int_{0}^{T}\left\|\left(P_{h} p-\bar{p}\right)(\tau)\right\|_{0}^{2} d \tau+\frac{\alpha^{2}}{2} \int_{0}^{T}\|(\tilde{P} \mathbf{u}-\overline{\mathbf{u}})(\tau)\|_{1}^{2} d \tau \\
& +\frac{1}{2} \int_{0}^{T}\left\|\left(p-P_{h} p\right)_{t}(\tau)\right\|_{0}^{2} d \tau+\frac{\alpha^{2}}{4 \epsilon_{2}}\left\|\left(p-P_{h} p\right)(T)\right\|_{0}^{2} \\
& +\frac{c_{0}}{2} \int_{0}^{T}\left\|\left(p-P_{h} p\right)_{t}(\tau)\right\|_{0}^{2} d \tau+\frac{c_{0}}{2} \int_{0}^{T}\left\|\left(P_{h} p-\bar{p}\right)(\tau)\right\|_{0}^{2} d \tau .
\end{aligned}
$$

Using Gronwall's inequality and combining constants on the right hand side of (40), we obtain

$$
\begin{array}{r}
\left(\frac{1}{2} C_{\text {coer }}-\epsilon_{2}\right)\|(\tilde{P} \mathbf{u}-\overline{\mathbf{u}})(T)\|_{1}^{2}+\frac{\gamma_{c}}{2}\left\|\left(P_{h} p-\bar{p}\right)(T)\right\|_{0}^{2} \\
+\left(\mu_{f} \zeta-\epsilon_{1}\right) \int_{0}^{T}\left\|\left(\Pi_{h} \mathbf{z}-\overline{\mathbf{z}}\right)(\tau)\right\|_{0}^{2} d \tau \\
\leq C\left[\int_{0}^{T}\|(\mathbf{u}-\tilde{P} \mathbf{u})(\tau)\|_{1}^{2} d \tau+\int_{0}^{T}\left\|\left(\mathbf{z}-\Pi_{h} \mathbf{z}\right)(\tau)\right\|_{0}^{2} d \tau\right. \\
+\int_{0}^{T}\left\|\left(p-P_{h} p\right)(\tau)\right\|_{0}^{2} d \tau+\int_{0}^{T}\left\|(\mathbf{u}-\tilde{P} \mathbf{u})_{t}(\tau)\right\|_{1}^{2} d \tau \\
\left.+\int_{0}^{T}\left\|\left(p-P_{h} p\right)_{t}(\tau)\right\|_{0}^{2} d \tau\right] .
\end{array}
$$

We conclude the proof using the approximation properties (7), (9) and (11). 
Combining the estimate given by Theorem 1, the triangle inequality and the interpolation estimates (7), (9) and (11), yields the following convergence result.

Theorem 2. Let $(\mathbf{u}, p, \mathbf{z})$ be the solution of (2)-(4) and $(\overline{\mathbf{u}}, \bar{p}, \overline{\mathbf{z}})$ be the solution of (16)-(18). Then

$$
\|\mathbf{u}-\overline{\mathbf{u}}\|_{L^{\infty}\left(H^{1}\right)}^{2}+\|p-\bar{p}\|_{L^{\infty}\left(L^{2}\right)}^{2}+\|\mathbf{z}-\overline{\mathbf{z}}\|_{L^{2}\left(L^{2}\right)}^{2} \leq C\left(H^{2 r}+h^{2 k+2}\right),
$$

where $C$ depends on the model parameters, $T, \zeta, L, \gamma_{c}, C_{c o e r}, p, p_{t}, \mathbf{u}, \mathbf{u}_{t}$ and on $M$ being sufficiently large but is not dependent on $H$ and $h$.

Corollary 1. Under the conditions of Theorem 2, the method is stable.

Proof Using the triangle inequality we get

$$
\|\overline{\mathbf{u}}\|_{L^{\infty}\left(H^{1}\right)} \leq\|\mathbf{u}-\overline{\mathbf{u}}\|_{L^{\infty}\left(H^{1}\right)}+\|\mathbf{u}\|_{L^{\infty}\left(H^{1}\right)} .
$$

From Theorem 2 and writing analogous inequalities for $\bar{p}$ and $\overline{\mathbf{z}}$, the result is immediate.

\section{Fully discrete approximation}

In this section we formulate the fully-discrete method by introducing the time discretization. For that purpose we use the Theta method.

Let $\Delta t=T / N$, where $N$ denotes the number of time steps and $t_{j}=j \Delta t$. We use the following notation $g_{j}=g\left(., t_{j}\right), g_{j, \theta}=\frac{1}{2}(1+\theta) g_{j+1}+\frac{1}{2}(1-\theta) g_{j}$.

The fully discrete method becomes: find $\overline{\mathbf{u}}_{j} \in \overline{\mathbf{u}}_{d, j}+\mathbf{V}_{h, 0}, \bar{p}_{j} \in W_{h}, \overline{\mathbf{z}}_{j} \in$ $\overline{\mathbf{z}}_{d, j}+\mathbf{V}_{h, 0}$ such that

$$
\begin{aligned}
a_{\mathbf{u}}\left(\overline{\mathbf{u}}_{j, \theta}, \mathbf{v}\right)-\alpha\left(\bar{p}_{j, \theta}, \nabla \cdot \mathbf{v}\right) & =\ell_{1 j, \theta}(\mathbf{v}), \\
\left(c_{0} \frac{\bar{p}_{j+1}-\bar{p}_{j}}{\Delta t}, w\right)+\alpha\left(\nabla \cdot \frac{\overline{\mathbf{u}}_{j+1}-\overline{\mathbf{u}}_{j}}{\Delta t}, w\right)+\left(\nabla \cdot \overline{\mathbf{z}}_{j, \theta}, w\right) & =\ell_{2 j, \theta}(w), \\
\mu_{f}\left(K^{-1}\left(\mathcal{M}\left(\bar{\sigma}_{m}\right)\right)_{j, \theta} \overline{\mathbf{z}}_{j, \theta}, \mathbf{s}\right)-\left(\bar{p}_{j, \theta}, \nabla \cdot \mathbf{s}\right) & =\ell_{3 j, \theta}(\mathbf{s})
\end{aligned}
$$

for all $(\mathbf{v}, w, \mathbf{s}) \in\left(\mathbf{V}_{h, 0}, W_{h}, \mathbf{S}_{h, 0}\right)$.

The time discretization corresponds to the backward Euler method if $\theta=1$, and to the Crank-Nicolson method if $\theta=0$. 
4.1. Discrete in time a priori error estimate. We assume the following regularity for the weak solution of the problem $(2)-(4): \mathbf{u} \in W^{2, \infty}([0, T]$; $\left.\left(H^{r+1}(\Omega)\right)^{d}\right), p \in W^{2, \infty}\left([0, T] ; H^{k+1}(\Omega)\right), \mathbf{z} \in L^{2}\left([0, T] ;\left(H^{k+1}(\Omega)\right)^{d}\right)$.

The following properties are well known:

$$
\frac{p_{j+1}-p_{j}}{\Delta t}=p_{t}\left(\mathbf{x}, t_{j, \theta}\right)+\Delta t \rho_{p, j, \theta}, \quad \forall \mathbf{x} \in \Omega
$$

with

$$
\left\|\rho_{p, j, \theta}\right\|_{0} \leq C\left\|p_{t t}\right\|_{L^{\infty}\left(\left(t_{j}, t_{j+1}\right), H^{1}\right)}
$$

and

$$
\frac{\mathbf{u}_{j+1}-\mathbf{u}_{j}}{\Delta t}=\mathbf{u}_{t}\left(\mathbf{x}, t_{j, \theta}\right)+\Delta t \rho_{\mathbf{u}, j, \theta}, \quad \forall \mathbf{x} \in \Omega
$$

with

$$
\left\|\rho_{\mathbf{u}, j, \theta}\right\|_{0} \leq C\left\|\mathbf{u}_{t t}\right\|_{L^{\infty}\left(\left(t_{j}, t_{j+1}\right), H^{1}\right)}
$$

where $\rho_{p}$ and $\rho_{\mathbf{u}}$ denote dependent on time derivatives of $p$ and $\mathbf{u}$, respectively. In the particular case of $\theta=0$, we also have

$$
\left\|\rho_{p, j, \theta}\right\|_{0} \leq \Delta t C\left\|p_{t t t}\right\|_{L^{\infty}\left(\left(t_{j}, t_{j+1}\right), H^{1}\right)},
$$

and

$$
\left\|\rho_{\mathbf{u}, j, \theta}\right\|_{0} \leq \Delta t C\left\|\mathbf{u}_{t t t}\right\|_{L^{\infty}\left(\left(t_{j}, t_{j+1}\right), H^{1}\right)} .
$$

Theorem 3. Let $(\mathbf{u}, p, \mathbf{z})$ be the solution of (2)-(4) and $(\overline{\mathbf{u}}, \bar{p}, \overline{\mathbf{z}})$ be the solution of (42)-(44). Then, if $\Delta t$ small enough, the exists $C>0$ such that $\|\tilde{P} \mathbf{u}-\overline{\mathbf{u}}\|_{L^{\infty}\left(H^{1}\right)}^{2}+\left\|P_{h} p-\bar{p}\right\|_{L^{\infty}\left(L^{2}\right)}^{2}+\left\|\Pi_{h} \mathbf{z}-\overline{\mathbf{z}}\right\|_{L^{2}\left(L^{2}\right)}^{2} \leq C\left(H^{2 r}+h^{2 k+2}\right)+\mathcal{O}\left(\Delta t^{2}\right)$,

where $C$ depends on the model parameters, $T, \zeta, L, \gamma_{c}, C_{c o e r}, p, p_{t}, \mathbf{u}, \mathbf{u}_{t}$ and on $M$ being sufficiently large but is not dependent on $H, h$ and $\Delta t$.

Proof For simplicity, let us assume that $\mathbf{u}_{0}, \mathbf{u}_{d} \in \mathbf{V}_{H}, p_{0} \in W_{h}$ and $\mathbf{z}_{d} \in \mathbf{S}_{h}$. This assumption does not affect the rate of convergence.

From (3), using (45) and (47) at time $t=t_{j, \theta}$, we obtain

$$
\begin{array}{r}
\left(c_{0} \frac{p_{j+1}-p_{j}}{\Delta t}, w\right)+\alpha\left(\nabla \cdot \frac{\mathbf{u}_{j+1}-\mathbf{u}_{j}}{\Delta t}, w\right)+\left(\nabla \cdot \mathbf{z}_{j, \theta}, w\right) \\
=\ell_{2, j, \theta}(w)-c_{0} \Delta t\left(\rho_{p, j, \theta}, w\right)-\alpha \Delta t\left(\nabla \cdot \rho_{\mathbf{u}, j, \theta}, w\right) .
\end{array}
$$

Subtracting (42)-(44) from (2)-(4) and using the above equality, we have

$$
a_{\mathbf{u}}\left(\mathbf{u}_{j, \theta}-\overline{\mathbf{u}}_{j, \theta}, \mathbf{v}\right)-\alpha\left(p_{j, \theta}-\bar{p}_{j, \theta}, \nabla \cdot \mathbf{v}\right)=0
$$




$$
\begin{array}{r}
\left(c_{0} \frac{\left(p_{j+1}-\bar{p}_{j+1}\right)-\left(p_{j}-\bar{p}_{j}\right)}{\Delta t}, w\right)+\alpha\left(\nabla \cdot \frac{\left(\mathbf{u}_{j+1}-\overline{\mathbf{u}}_{j+1}\right)-\left(\mathbf{u}_{j}-\overline{\mathbf{u}}_{j}\right)}{\Delta t}, w\right) \\
+\left(\nabla \cdot\left(\mathbf{z}_{j, \theta}-\overline{\mathbf{z}}_{j, \theta}\right), w\right)=-c_{0} \Delta t\left(\rho_{p, j, \theta}, w\right)-\alpha \Delta t\left(\nabla \cdot \rho_{\mathbf{u}, j, \theta}, w\right)(53)
\end{array}
$$

and

$$
\mu_{f}\left(\left(K^{-1}\left(\sigma_{m}\right)_{j, \theta} \mathbf{z}_{j, \theta}-K^{-1}\left(\mathcal{M}\left(\bar{\sigma}_{m}\right)\right)_{j, \theta}\right) \overline{\mathbf{z}}_{j, \theta}, \mathbf{s}\right)-\left(\bar{p}_{j, \theta}, \nabla \cdot \mathbf{s}\right)=0 .
$$

Let $\mathbf{v}=\frac{(\tilde{P} \mathbf{u}-\overline{\mathbf{u}})_{j+1}-(\tilde{P} \mathbf{u}-\overline{\mathbf{u}})_{j}}{\Delta t}, w=\left(P_{h} p-\bar{p}\right)_{j, \theta}$ and $\mathbf{s}=\left(\Pi_{h} \mathbf{z}-\overline{\mathbf{z}}\right)_{j, \theta}$.

From (52), using the properties of the elliptic projection, we derive

$$
\begin{aligned}
& a_{\mathbf{u}}\left((\tilde{P} \mathbf{u}-\overline{\mathbf{u}})_{j, \theta}, \frac{(\tilde{P} \mathbf{u}-\overline{\mathbf{u}})_{j+1}-(\tilde{P} \mathbf{u}-\overline{\mathbf{u}})_{j}}{\Delta t}\right) \\
& -\alpha\left(\left(p-P_{h} p\right)_{j, \theta}, \nabla \cdot \frac{(\tilde{P} \mathbf{u}-\overline{\mathbf{u}})_{j+1}-(\tilde{P} \mathbf{u}-\overline{\mathbf{u}})_{j}}{\Delta t}\right) \\
& -\alpha\left(\left(P_{h} p-\bar{p}\right)_{j, \theta}, \nabla \cdot \frac{(\tilde{P} \mathbf{u}-\overline{\mathbf{u}})_{j+1}-(\tilde{P} \mathbf{u}-\overline{\mathbf{u}})_{j}}{\Delta t}\right)=0 .
\end{aligned}
$$

Summing (53) and (54) and noting orthogonality relationships, we have

$$
\begin{aligned}
& \left(c_{0} \frac{\left(P_{h} p-\bar{p}\right)_{j+1}-\left(P_{h} p-\bar{p}\right)_{j}}{\Delta t},\left(P_{h} p-\bar{p}\right)_{j, \theta}\right) \\
& +\alpha\left(\nabla \cdot \frac{(\mathbf{u}-\tilde{P} \mathbf{u})_{j+1}-(\mathbf{u}-\tilde{P} \mathbf{u})_{j}}{\Delta t},\left(P_{h} p-\bar{p}\right)_{j, \theta}\right) \\
& +\alpha\left(\nabla \cdot \frac{(\tilde{P} \mathbf{u}-\overline{\mathbf{u}})_{j+1}-(\tilde{P} \mathbf{u}-\overline{\mathbf{u}})_{j}}{\Delta t},\left(P_{h} p-\bar{p}\right)_{j, \theta}\right) \\
& +\mu_{f}\left(K^{-1}\left(\sigma_{m}\right)_{j, \theta}\left(\mathbf{z}-\Pi_{h} \mathbf{z}\right)_{j, \theta},\left(\Pi_{h} \mathbf{z}-\overline{\mathbf{z}}\right)_{j, \theta}\right) \\
& +\mu_{f}\left(\left(K^{-1}\left(\sigma_{m}\right)_{j, \theta}-K^{-1}\left(\mathcal{M}\left(\bar{\sigma}_{m}\right)\right)_{j, \theta}\right) \Pi_{h} \mathbf{z}_{j, \theta},\left(\Pi_{h} \mathbf{z}-\overline{\mathbf{z}}\right)_{j, \theta}\right) \\
& +\mu_{f}\left(K^{-1}\left(\mathcal{M}\left(\bar{\sigma}_{m}\right)\right)_{j, \theta}\left(\Pi_{h} \mathbf{z}-\overline{\mathbf{z}}\right)_{j, \theta},\left(\Pi_{h} \mathbf{z}-\overline{\mathbf{z}}\right)_{j, \theta}\right) \\
& =-c_{0} \Delta t\left(\rho_{p, j, \theta},\left(P_{h} p-\bar{p}\right)_{j, \theta}\right)-\alpha \Delta t\left(\nabla \cdot \rho_{\mathbf{u}, j, \theta},\left(P_{h} p-\bar{p}\right)_{j, \theta}\right) .
\end{aligned}
$$

Summing (55) and (56), we obtain

$$
\begin{aligned}
& a_{\mathbf{u}}\left((\tilde{P} \mathbf{u}-\overline{\mathbf{u}})_{j, \theta}, \frac{(\tilde{P} \mathbf{u}-\overline{\mathbf{u}})_{j+1}-(\tilde{P} \mathbf{u}-\overline{\mathbf{u}})_{j}}{\Delta t}\right) \\
& +\left(c_{0}\left(P_{h} p-\bar{p}\right)_{j, \theta}, \frac{\left(P_{h} p-\bar{p}\right)_{j+1}-\left(P_{h} p-\bar{p}\right)_{j}}{\Delta t}\right) \\
& +\mu_{f}\left(K^{-1}\left(\mathcal{M}\left(\bar{\sigma}_{m}\right)\right)_{j, \theta}\left(\Pi_{h} \mathbf{z}-\overline{\mathbf{z}}\right)_{j, \theta},\left(\Pi_{h} \mathbf{z}-\overline{\mathbf{z}}\right)_{j, \theta}\right)
\end{aligned}
$$




$$
\begin{aligned}
& =\alpha\left(\nabla \cdot \frac{(\tilde{P} \mathbf{u}-\overline{\mathbf{u}})_{j+1}-(\tilde{P} \mathbf{u}-\overline{\mathbf{u}})_{j}}{\Delta t},\left(p-P_{h} p\right)_{j, \theta}\right) \\
& -\alpha\left(\nabla \cdot \frac{(\mathbf{u}-\tilde{P} \mathbf{u})_{j+1}-(\mathbf{u}-\tilde{P} \mathbf{u})_{j}}{\Delta t},\left(P_{h} p-\bar{p}\right)_{j, \theta}\right) \\
& -\mu_{f}\left(K^{-1}\left(\sigma_{m}\right)_{j, \theta}\left(\mathbf{z}-\Pi_{h} \mathbf{z}\right)_{j, \theta},\left(\Pi_{h} \mathbf{z}-\overline{\mathbf{z}}\right)_{j, \theta}\right) \\
& -\mu_{f}\left(\left(K^{-1}\left(\sigma_{m}\right)_{j, \theta}-K^{-1}\left(\mathcal{M}\left(\bar{\sigma}_{m}\right)\right)_{j, \theta}\right) \Pi_{h} \mathbf{z}_{j, \theta},\left(\Pi_{h} \mathbf{z}-\overline{\mathbf{z}}\right)_{j, \theta}\right) \\
& -c_{0} \Delta t\left(\rho_{p, j, \theta},\left(P_{h} p-\bar{p}\right)_{j, \theta}\right)-\alpha \Delta t\left(\nabla \cdot \rho_{\mathbf{u}, j, \theta},\left(P_{h} p-\bar{p}\right)_{j, \theta}\right) .
\end{aligned}
$$

In order to provide the desire bounds we start by deducing the following inequality

$$
\begin{aligned}
& a_{\mathbf{u}}\left((\tilde{P} \mathbf{u}-\overline{\mathbf{u}})_{j, \theta}, \frac{(\tilde{P} \mathbf{u}-\overline{\mathbf{u}})_{j+1}-(\tilde{P} \mathbf{u}-\overline{\mathbf{u}})_{j}}{\Delta t}\right) \\
= & \frac{1+\theta}{2 \Delta t} a_{\mathbf{u}}\left((\tilde{P} \mathbf{u}-\overline{\mathbf{u}})_{j+1},(\tilde{P} \mathbf{u}-\overline{\mathbf{u}})_{j+1}\right) \\
& -\frac{1+\theta}{2 \Delta t} a_{\mathbf{u}}\left((\tilde{P} \mathbf{u}-\overline{\mathbf{u}})_{j+1},(\tilde{P} \mathbf{u}-\overline{\mathbf{u}})_{j}\right) \\
& +\frac{1-\theta}{2 \Delta t} a_{\mathbf{u}}\left((\tilde{P} \mathbf{u}-\overline{\mathbf{u}})_{j},(\tilde{P} \mathbf{u}-\overline{\mathbf{u}})_{j+1}\right) \\
& -\frac{1-\theta}{2 \Delta t} a_{\mathbf{u}}\left((\tilde{P} \mathbf{u}-\overline{\mathbf{u}})_{j},(\tilde{P} \mathbf{u}-\overline{\mathbf{u}})_{j}\right) \\
= & \frac{1}{2 \Delta t}\left\|(\tilde{P} \mathbf{u}-\overline{\mathbf{u}})_{j+1}\right\|_{a_{\mathbf{u}}}-\frac{1}{2 \Delta t}\left\|(\tilde{P} \mathbf{u}-\overline{\mathbf{u}})_{j}\right\|_{a_{\mathbf{u}}} \\
& +\frac{\theta}{2 \Delta t}\left\|(\tilde{P} \mathbf{u}-\overline{\mathbf{u}})_{j+1}-(\tilde{P} \mathbf{u}-\overline{\mathbf{u}})_{j}\right\|_{a_{\mathbf{u}}} \\
\geq & \frac{1}{2 \Delta t}\left(\left\|(\tilde{P} \mathbf{u}-\overline{\mathbf{u}})_{j+1}\right\|_{a_{\mathbf{u}}}-\left\|(\tilde{P} \mathbf{u}-\overline{\mathbf{u}})_{j}\right\|_{a_{\mathbf{u}}}\right) .
\end{aligned}
$$

Likewise, we have

$$
\begin{aligned}
& \left(\left(P_{h} p-\bar{p}\right)_{j, \theta}, \frac{\left(P_{h} p-\bar{p}\right)_{j+1}-\left(P_{h} p-\bar{p}\right)_{j}}{\Delta t}\right) \\
\geq & \frac{1}{2 \Delta t}\left(\left\|\left(P_{h} p-\bar{p}\right)_{j+1}\right\|_{0}-\left\|\left(P_{h} p-\bar{p}\right)_{j}\right\|_{0}\right) .
\end{aligned}
$$

In the next bound we take in account that $\left\|(\tilde{P} \mathbf{u}-\overline{\mathbf{u}})_{\mid t=0}\right\|_{a_{\mathbf{u}}}=0, \|\left(P_{h} p-\right.$ $\bar{p})_{\mid t=0} \|_{0}=0$, and we use (6), (14), (58) and (59). Multiplying (57) by $2 \Delta t$ 
and summing from 0 to $N-1$, we obtain

$$
\begin{array}{r}
\left\|(\tilde{P} \mathbf{u}-\overline{\mathbf{u}})_{N}\right\|_{a_{\mathbf{u}}}^{2}+\gamma_{c}\left\|\left(P_{h} p-\bar{p}\right)_{N}\right\|_{0}^{2}+2 \mu_{f} \zeta \sum_{j=0}^{N-1}\left\|\left(\Pi_{h} \mathbf{z}-\overline{\mathbf{z}}\right)_{j, \theta}\right\|_{0}^{2} \Delta t \\
\leq \psi_{1}+\psi_{2}+\psi_{3}+\psi_{4}+\psi_{5}+\psi_{6}
\end{array}
$$

where

$$
\begin{gathered}
\psi_{1}=2 \alpha \sum_{j=0}^{N-1}\left(\nabla \cdot \frac{(\tilde{P} \mathbf{u}-\overline{\mathbf{u}})_{j+1}-(\tilde{P} \mathbf{u}-\overline{\mathbf{u}})_{j}}{\Delta t},\left(p-P_{h} p\right)_{j, \theta}\right) \Delta t \\
\psi_{2}=-2 \alpha \sum_{j=0}^{N-1}\left(\nabla \cdot \frac{(\mathbf{u}-\tilde{P} \mathbf{u})_{j+1}-(\mathbf{u}-\tilde{P} \mathbf{u})_{j}}{\Delta t},\left(P_{h} p-\bar{p}\right)_{j, \theta}\right) \Delta t, \\
\psi_{3}=-2 \mu_{f} \sum_{j=0}^{N-1}\left(K^{-1}\left(\sigma_{m}\right)_{j, \theta}\left(\mathbf{z}-\Pi_{h} \mathbf{z}\right)_{j, \theta},\left(\Pi_{h} \mathbf{z}-\overline{\mathbf{z}}\right)_{j, \theta}\right) \Delta t \\
-2 \mu_{f} \sum_{j=0}^{N-1}\left(\left(K^{-1}\left(\sigma_{m}\right)_{j, \theta}-K^{-1}\left(\mathcal{M}\left(\bar{\sigma}_{m}\right)\right)_{j, \theta}\right) \Pi_{h} \mathbf{z}_{j, \theta},\left(\Pi_{h} \mathbf{z}-\overline{\mathbf{z}}\right)_{j, \theta}\right) \Delta t \\
\psi_{4}=-2 c_{0} \sum_{j=0}^{N-1} \Delta t\left(\rho_{p, j, \theta},\left(P_{h} p-\bar{p}\right)_{j, \theta}\right)_{\Delta t} \\
\psi_{5}=-2 \alpha \sum_{j=0}^{N-1} \Delta t\left(\nabla \cdot \rho_{\mathbf{u}, j, \theta},\left(P_{h} p-\bar{p}\right)_{j, \theta}\right) \Delta t .
\end{gathered}
$$

Summing by parts and noting that $\left\|(\tilde{P} \mathbf{u}-\overline{\mathbf{u}})_{\mid t=0}\right\|_{1}=0$, we see that

$$
\begin{aligned}
\psi_{1}= & 2 \alpha\left(\nabla \cdot(\tilde{P} \mathbf{u}-\overline{\mathbf{u}})_{N},\left(p-P_{h} p\right)_{N, \theta}\right) \\
& -2 \alpha \sum_{j=0}^{N-1}\left(\nabla \cdot(\tilde{P} \mathbf{u}-\overline{\mathbf{u}})_{j+1}, \frac{\left(p-P_{h} p\right)_{j+1, \theta}-\left(p-P_{h} p\right)_{j, \theta}}{\Delta t}\right) \Delta t \\
\leq & 2 \alpha\left\|\nabla \cdot(\tilde{P} \mathbf{u}-\overline{\mathbf{u}})_{N}\right\|_{0}\left\|\left(p-P_{h} p\right)_{N, \theta}\right\|_{0} \\
& +2 \alpha \Delta t \sum_{j=0}^{N-1}\left\|\nabla \cdot(\tilde{P} \mathbf{u}-\overline{\mathbf{u}})_{j+1}\right\|_{0}\left\|\frac{\left(p-P_{h} p\right)_{j+1, \theta}-\left(p-P_{h} p\right)_{j, \theta}}{\Delta t}\right\|_{0} .
\end{aligned}
$$


Using (46), we obtain

$$
\begin{aligned}
\psi_{1} \leq & \epsilon_{1}\left\|(\tilde{P} \mathbf{u}-\overline{\mathbf{u}})_{N}\right\|_{1}^{2}+\frac{\alpha^{2}}{4 \epsilon_{1}}\left\|\left(p-P_{h} p\right)_{N, \theta}\right\|_{0}^{2}+\alpha \Delta t \sum_{j=0}^{N}\left\|(\tilde{P} \mathbf{u}-\overline{\mathbf{u}})_{j}\right\|_{1}^{2} \\
& +2 \alpha \Delta t \sum_{j=0}^{N}\left\|\left(\left(p-P_{h} p\right)_{j}\right)_{t}\right\|_{0}^{2}+2 \alpha \Delta t^{2}\left\|\rho_{p, j, \theta}\right\|_{0}^{2}
\end{aligned}
$$

where $\epsilon_{1}$ is an arbitrary small constant. Likewise, approximating the difference with a derivative, yields the bound

$$
\psi_{2} \leq 2 \alpha \Delta t \sum_{j=0}^{N}\left\|\left((\mathbf{u}-\tilde{P} \mathbf{u})_{j, \theta}\right)_{t}\right\|_{1}^{2}+\alpha \Delta t \sum_{j=0}^{N}\left\|\left(P_{h} p-\bar{p}\right)_{j}\right\|_{0}^{2}+C \Delta t^{2} .
$$

Using (12) and (15), it follows that

$$
\begin{aligned}
\psi_{3} \leq & 2 \mu_{f} \sum_{j=0}^{N-1}\left\|K^{-1}\left(\sigma_{m}\right)_{j, \theta}\right\|_{\infty}\left\|\left(\mathbf{z}-\Pi_{h} \mathbf{z}\right)_{j, \theta}\right\|_{0}\left\|\left(\Pi_{h} \mathbf{z}-\overline{\mathbf{z}}\right)_{j, \theta}\right\|_{0} \Delta t \\
& +2 \mu_{f} \sum_{j=0}^{N-1} \|\left(K^{-1}\left(\sigma_{m}\right)_{j, \theta}-K^{-1}\left(\mathcal{M}\left(\bar{\sigma}_{m}\right)\right)_{j, \theta}\left\|_{0}\right\| \Pi_{h} \mathbf{z}_{j, \theta}\left\|_{\infty}\right\|\left(\Pi_{h} \mathbf{z}-\overline{\mathbf{z}}\right)_{j, \theta} \|_{0} \Delta t\right. \\
\leq & \frac{\mu_{f}^{2}}{\epsilon_{2}} \sum_{j=0}^{N-1}\left\|K^{-1}\left(\sigma_{m}\right)_{j, \theta}\right\|_{\infty}^{2}\left\|\left(\mathbf{z}-\Pi_{h} \mathbf{z}\right)_{j, \theta}\right\|_{0}^{2} \Delta t+\epsilon_{2} \sum_{j=0}^{N-1}\left\|\left(\Pi_{h} \mathbf{z}-\overline{\mathbf{z}}\right)_{j, \theta}\right\|_{0}^{2} \Delta t \\
& +\frac{L}{\epsilon_{2}} \sum_{j=0}^{N-1}\left\|\left(\sigma_{m}\right)_{j, \theta}-\left(\bar{\sigma}_{m}\right)_{j, \theta}\right\|_{0}^{2}\left\|\Pi_{h} \mathbf{z}_{j, \theta}\right\|_{\infty}^{2} \Delta t+\epsilon_{2} \sum_{j=0}^{N-1}\left\|\left(\Pi_{h} \mathbf{z}-\overline{\mathbf{z}}\right)_{j, \theta}\right\|_{0}^{2} \Delta t \\
\leq & \frac{\mu_{f}^{2}}{\epsilon_{2}} \sum_{j=0}^{N-1}\left\|K^{-1}\left(\sigma_{m}\right)_{j, \theta}\right\|_{\infty}^{2}\left\|\left(\mathbf{z}-\Pi_{h} \mathbf{z}\right)_{j, \theta}\right\|_{0}^{2} \Delta t+2 \epsilon_{2} \sum_{j=0}^{N-1}\left\|\left(\Pi_{h} \mathbf{z}-\overline{\mathbf{z}}\right)_{j, \theta}\right\|_{0}^{2} \Delta t \\
& +\frac{L}{2 \epsilon_{2}}(2 \mu+2 \lambda)^{2} \sum_{j=0}^{N-1}\left\|\mathbf{u}_{j, \theta}-\tilde{P}_{\mathbf{u}_{j, \theta}}\right\|_{1}^{2}\left\|\Pi_{h} \mathbf{z}_{j, \theta}\right\|_{\infty}^{2} \Delta t \\
& +\frac{L}{2 \epsilon_{2}}(2 \mu+2 \lambda)^{2} \sum_{j=0}^{N-1}\left\|\tilde{P} \mathbf{u}_{j, \theta}-\overline{\mathbf{u}}_{j, \theta}\right\|_{1}^{2}\left\|\Pi_{h} \mathbf{z}_{j, \theta}\right\|_{\infty}^{2} \Delta t
\end{aligned}
$$

where $\epsilon_{2}$ is an arbitrary small constant. 
By the Cauchy-Schwarz inequality we find the relations

$$
\begin{aligned}
& \psi_{4} \leq c_{0} \sum_{j=0}^{N-1} \Delta t^{2}\left\|\rho_{p, j, \theta}\right\|_{0}^{2}+c_{0} \sum_{j=0}^{N-1} \Delta t^{2}\left\|\left(P_{h} p-\bar{p}\right)_{j, \theta}\right\|_{0}^{2}, \\
& \psi_{5} \leq \alpha \sum_{j=0}^{N-1} \Delta t^{2}\left\|\rho_{\mathbf{u}, j, \theta}\right\|_{1}^{2}+\alpha \sum_{j=0}^{N-1} \Delta t^{2}\left\|\left(P_{h} p-\bar{p}\right)_{j, \theta}\right\|_{0}^{2} .
\end{aligned}
$$

Using the above bounds, the coercivity of $a_{\mathbf{u}}$ and the assumptions (5) and (6), we obtain the inequality

$$
\begin{aligned}
& \left(C_{\text {coer }}-\epsilon_{1}\right)\left\|(\tilde{P} \mathbf{u}-\overline{\mathbf{u}})_{N}\right\|_{1}^{2}+\gamma_{c}\left\|\left(P_{h} p-\bar{p}\right)_{N}\right\|_{0}^{2} \\
& +2\left(\mu_{f} \zeta-\epsilon_{2}\right) \sum_{j=0}^{N-1}\left\|\left(\Pi_{h} \mathbf{z}-\overline{\mathbf{z}}\right)_{j, \theta}\right\|_{0}^{2} \Delta t \\
& \leq C\left(\Delta t \sum_{j=0}^{N-1}\left\|(\tilde{P} \mathbf{u}-\overline{\mathbf{u}})_{j, \theta}\right\|_{1}^{2}+\Delta t \sum_{j=0}^{N-1}\left\|\left(P_{h} p-\bar{p}\right)_{j}\right\|_{0}^{2}+\left\|\left(p-P_{h} p\right)_{N, \theta}\right\|_{0}^{2}\right. \\
& +\Delta t \sum_{j=0}^{N}\left\|\left(\left(p-P_{h} p\right)_{j}\right)_{t}\right\|_{0}^{2}+\Delta t \sum_{j=0}^{N}\left\|\left((\tilde{P} \mathbf{u}-\overline{\mathbf{u}})_{j}\right)_{t}\right\|_{1}^{2} \\
& \left.+\Delta t \sum_{j=0}^{N-1}\left\|\left(\mathbf{z}-\Pi_{h} \mathbf{z}\right)_{j, \theta}\right\|_{0}^{2}\right)+\mathcal{O}\left(\Delta t^{2}\right) .
\end{aligned}
$$

For $\Delta t$ sufficiently small, we may apply the discrete version of Gronwall's lemma (see e.g. [5]), in order to obtain (51).

If we consider the Crank-Nicolson method $(\theta=0)$ in $(42)-(44)$ and if we assume the regularity $\mathbf{u} \in W^{3, \infty}\left([0, T] ;\left(H^{r+1}(\Omega)\right)^{d}\right)$ and $p \in W^{3, \infty}([0, T]$; $\left.\left(H^{k+1}(\Omega)\right)^{d}\right)$, then we can replace $(51)$ by

$\|\tilde{P} \mathbf{u}-\overline{\mathbf{u}}\|_{L^{\infty}\left(H^{1}\right)}^{2}+\left\|P_{h} p-\bar{p}\right\|_{L^{\infty}\left(L^{2}\right)}^{2}+\left\|\Pi_{h} \mathbf{z}-\overline{\mathbf{z}}\right\|_{L^{2}\left(L^{2}\right)}^{2} \leq C\left(H^{2 r}+h^{2 k+2}\right)+\mathcal{O}\left(\Delta t^{4}\right)$.

The convergence result in the next theorem is obtained combining the interpolation estimates and the auxiliary estimates by the triangle inequality.

Theorem 4. Let $(\mathbf{u}, p, \mathbf{z})$ be the solution of (2)-(4) and $(\overline{\mathbf{u}}, \bar{p}, \overline{\mathbf{z}})$ be the solution of (42)-(44). Then, if $\Delta t$ small enough, there exists $C>0$ such that

$$
\|\mathbf{u}-\overline{\mathbf{u}}\|_{L^{\infty}\left(H^{1}\right)}^{2}+\|p-\bar{p}\|_{L^{\infty}\left(L^{2}\right)}^{2}+\|\mathbf{z}-\overline{\mathbf{z}}\|_{L^{2}\left(L^{2}\right)}^{2} \leq C\left(H^{2 r}+h^{2 k+2}\right)+\mathcal{O}\left(\Delta t^{2}\right),
$$

where $C$ depends on on the model parameters, $T, \zeta, L, \gamma_{c}, C_{c o e r}, p, p_{t}, \mathbf{u}, \mathbf{u}_{t}$ and on $M$ being sufficiently large but is not dependent on $H, h$ and $\Delta t$. 
If we use the Crank-Nicolson method, under the mentioned regularity conditions for this case, holds

$$
\|\mathbf{u}-\overline{\mathbf{u}}\|_{L^{\infty}\left(H^{1}\right)}^{2}+\|p-\bar{p}\|_{L^{\infty}\left(L^{2}\right)}^{2}+\|\mathbf{z}-\overline{\mathbf{z}}\|_{L^{2}\left(L^{2}\right)}^{2} \leq C\left(H^{2 r}+h^{2 k+2}\right)+\mathcal{O}\left(\Delta t^{4}\right) .
$$

\section{Numerical results}

For the numerical experiments we consider the Mandel's problem ([1],[8]). This example involves a poroelastic rectangular infinitely long slab, of extent $2 a$ in the $x$ direction and $2 b$ in the $y$ direction, sandwiched at the top and the bottom by two rigid plates. In the initial instant a force of magnitude $2 F$ is applied at the top and at the bottom plates, pointing to the slab. Since the plates are rigid then the slab remains in contact with the plates, that is, the vertical displacements at the top and bottom are uniform. The problem is symmetric about the $x$ and $y$ axes and we can reduce the computational domain to only the upper right quadrant $\Omega=(0, a) \times(0, b)$. Thus, the governing equations are the following:

$$
\begin{aligned}
-(\lambda+\mu) \nabla(\nabla \cdot \mathbf{u})-\mu \nabla^{2} \mathbf{u}+\alpha \nabla p & =0 \text { in } \Omega \times(0, T] \\
\frac{\partial}{\partial t}\left(c_{0} p+\alpha \nabla \cdot \mathbf{u}\right)-\frac{1}{\mu_{f}} \nabla \cdot K\left(\sigma_{m}\right) \nabla p & =0 \text { in } \Omega \times(0, T] \\
p & =0, x=a, t \in(0, T] \\
-\frac{1}{\mu_{f}} K\left(\sigma_{m}\right) \nabla p \cdot \eta & =0, x=0, y=0, y=b, t \in(0, T] \\
u_{x} & =0, x=0, t \in(0, T] \\
u_{y} & =0, y=0, t \in(0, T] \\
\frac{\partial u_{y}}{\partial x} & =0, y=b, t \in(0, T] \\
\tilde{\sigma} \eta & =(-F / a) \eta, y=b, t \in(0, T] \\
\tilde{\sigma} \eta & =0, x=0, x=a, y=0, t \in(0, T] \\
p(0) & =0 \text { in } \Omega,
\end{aligned}
$$

where $u_{x}$ and $u_{y}$ are the components of $\mathbf{u}$.

The analytical solution of the Mandel's model is available, in [1], for the linear model, where the permeability is considered constant. In this case, the numerical solution can be directly compared with the analytical solution, which motivates the use of the Mandel's model as a benchmark problem for testing numerical codes (e.g. [6], [7], [11]).

The optimal error estimates are obtained under the assumption of a sufficiently smooth analytical solution. For the Mandel's problem, as $t \rightarrow 0$, the pressure presents very large gradient, since $p(0)=0$ and immediately after the application of the force the pressure increases suddenly. For this reason, 
the regularity of the pressure might be limited. To overcome this limitation and for the purpose of illustrating numerically the error estimate of Theorem 2 , we use an initial time $t_{0}>0$.

In the numerical experiments we used the following data: $\Omega=(0,1) \times$ $(0,0.1), \mu_{f}=1, F=2, k=1, k_{b}=1$ and $t_{0}=5 e-5$. The other parameters, which are not listed above, are given by the expressions

$$
\begin{aligned}
\lambda & =(E \nu) /((1+\nu)(1-2 \nu)), \\
\mu & =E /(2(\nu+1)), \\
\alpha & =3\left(\nu_{u}-\nu\right) /\left(B(1-2 \nu)\left(1+\nu_{u}\right)\right), \\
c_{0} & =\left(9\left(\nu_{u}-\nu\right)\left(1-2 \nu_{u}\right)\right) /\left(2 \mu B^{2}(1-2 \nu)\left(1+\nu_{u}\right)^{2}\right),
\end{aligned}
$$

that also depend on the Skempton poro pressure coefficient $B$, the Young's modulus $E$, the drained Poisson ratio $\nu$ and the undrained Poisson ratio $\nu_{u}$. For this parameters we considered $B=0.8, E=10, \nu=0.2$ and $\nu_{u}=0.4$.

The implicit Euler scheme is used. To minimize the effects of the error produced by the time discretization, we choose the small time step $\Delta t=$ $1 e-8$.

We start the computation using the analytical exact solution as starting solution at the time $t_{0}$, considering $K=I$,

\begin{tabular}{|c||c|c||c|c|||c|c|}
\hline$H$ & $\|\mathbf{u}-\overline{\mathbf{u}}\|_{H^{1}}$ & rate & $\|p-\bar{p}\|_{L^{2}}$ & rate & $\|\mathbf{z}-\overline{\mathbf{z}}\|_{L^{2}}$ & rate \\
\hline $5.000 \mathrm{e}-2$ & $1.222 \mathrm{e}-3$ & 1.39 & $1.389 \mathrm{e}-2$ & 1.53 & $2.416 \mathrm{e}-1$ & 1.35 \\
$2.500 \mathrm{e}-2$ & $4.653 \mathrm{e}-4$ & 1.19 & $4.798 \mathrm{e}-3$ & 1.21 & $9.452 \mathrm{e}-2$ & 0.94 \\
$1.667 \mathrm{e}-2$ & $2.878 \mathrm{e}-4$ & 1.05 & $2.933 \mathrm{e}-3$ & 1.03 & $6.453 \mathrm{e}-2$ & 1.14 \\
$1.250 \mathrm{e}-2$ & $2.130 \mathrm{e}-4$ & 1.10 & $2.179 \mathrm{e}-3$ & 1.08 & $4.654 \mathrm{e}-2$ & 0.82 \\
$1.000 \mathrm{e}-2$ & $1.665 \mathrm{e}-4$ & 0.89 & $1.711 \mathrm{e}-3$ & 0.92 & $3.875 \mathrm{e}-2$ & 1.14 \\
$8.333 \mathrm{e}-3$ & $1.415 \mathrm{e}-4$ & - & $1.446 \mathrm{e}-3$ & - & $3.149 \mathrm{e}-2$ & - \\
\hline
\end{tabular}

TABLE 1. Convergence rates: linear problem, bilinear elements for $\mathbf{u}$, lowest order Raviart-Thomas space for $p$ and $\mathbf{z}$.

For numerical verification of our algorithms, we show the computed errors for the linear case, taking $K=k I$. The analytical solution of this problem can be found e.g. in [1].

For the nonlinear problem, we considered stress dependent permeability using the formula for the first type of rock, $K=K_{0} e^{-k_{b} \sigma_{m}}$, taking $K_{0}=0.9 I$.

A Newton iteration with the numerical solution of the previous time step as starting guess is used to resolve the nonlinearity. 


\begin{tabular}{|c||c|c||c|c||c|c|}
\hline$H$ & $\|\mathbf{u}-\overline{\mathbf{u}}\|_{H^{1}}$ & rate & $\|p-\bar{p}\|_{L^{2}}$ & rate & $\|\mathbf{z}-\overline{\mathbf{z}}\|_{L^{2}}$ & rate \\
\hline $5.000 \mathrm{e}-2$ & $1.247 \mathrm{e}-4$ & 2.35 & $1.247 \mathrm{e}-003$ & 2.44 & $8.407 \mathrm{e}-2$ & 3.05 \\
$2.500 \mathrm{e}-2$ & $2.440 \mathrm{e}-5$ & 2.14 & $2.306 \mathrm{e}-004$ & 2.08 & $1.016 \mathrm{e}-2$ & 2.30 \\
$1.667 \mathrm{e}-2$ & $1.025 \mathrm{e}-5$ & 2.33 & $9.929 \mathrm{e}-005$ & 2.38 & $3.997 \mathrm{e}-3$ & 2.05 \\
$1.250 \mathrm{e}-2$ & $5.242 \mathrm{e}-6$ & 1.73 & $5.002 \mathrm{e}-005$ & 1.71 & $2.213 \mathrm{e}-3$ & 2.43 \\
$1.000 \mathrm{e}-2$ & $3.565 \mathrm{e}-6$ & 2.26 & $3.414 \mathrm{e}-005$ & 2.24 & $1.288 \mathrm{e}-3$ & 1.60 \\
$8.333 \mathrm{e}-3$ & $2.361 \mathrm{e}-6$ & - & $2.268 \mathrm{e}-005$ & - & $9.614 \mathrm{e}-4$ & - \\
\hline
\end{tabular}

TABLE 2. Convergence rates: linear problem, biquadratic elements for $\mathbf{u}$, Raviart-Thomas space of order 1 for $p$ and $\mathbf{z}$.

\begin{tabular}{|c||c|c||c|c||c|c|}
\hline$H$ & $\|\mathbf{u}-\overline{\mathbf{u}}\|_{H^{1}}$ & rate & $\|p-\bar{p}\|_{L^{2}}$ & rate & $\|\mathbf{z}-\overline{\mathbf{z}}\|_{L^{2}}$ & rate \\
\hline $5.000 \mathrm{e}-2$ & $1.078 \mathrm{e}-3$ & 1.45 & $9.591 \mathrm{e}-3$ & 1.27 & $2.378 \mathrm{e}-1$ & 0.91 \\
$2.500 \mathrm{e}-2$ & $3.948 \mathrm{e}-4$ & 1.05 & $3.978 \mathrm{e}-3$ & 1.25 & $1.263 \mathrm{e}-1$ & 1.15 \\
$1.667 \mathrm{e}-2$ & $2.574 \mathrm{e}-4$ & 1.09 & $2.394 \mathrm{e}-3$ & 1.22 & $7.916 \mathrm{e}-2$ & 1.17 \\
$1.250 \mathrm{e}-2$ & $1.882 \mathrm{e}-4$ & 0.70 & $1.684 \mathrm{e}-3$ & 1.00 & $5.649 \mathrm{e}-2$ & 1.13 \\
$1.000 \mathrm{e}-2$ & $1.608 \mathrm{e}-4$ & 1.84 & $1.348 \mathrm{e}-3$ & 1.80 & $4.388 \mathrm{e}-2$ & 1.10 \\
$8.333 \mathrm{e}-3$ & $1.150 \mathrm{e}-4$ & - & $9.715 \mathrm{e}-4$ & - & $3.593 \mathrm{e}-2$ & - \\
\hline
\end{tabular}

TABLE 3. Convergence rates: nonlinear problem, bilinear elements for $\mathbf{u}$, lowest order Raviart-Thomas space for $p$ and $\mathbf{z}$.

\begin{tabular}{|c||c|c||c|c||c|c|}
\hline$H$ & $\|\mathbf{u}-\overline{\mathbf{u}}\|_{H^{1}}$ & rate & $\|p-\bar{p}\|_{L^{2}}$ & rate & $\|\mathbf{z}-\overline{\mathbf{z}}\|_{L^{2}}$ & rate \\
\hline $5.000 \mathrm{e}-2$ & $1.316 \mathrm{e}-4$ & 2.26 & $1.185 \mathrm{e}-3$ & 2.30 & $2.157 \mathrm{e}-1$ & 1.87 \\
$2.500 \mathrm{e}-2$ & $2.756 \mathrm{e}-5$ & 1.78 & $2.410 \mathrm{e}-4$ & 1.85 & $5.885 \mathrm{e}-2$ & 1.69 \\
$1.667 \mathrm{e}-2$ & $1.338 \mathrm{e}-5$ & 2.08 & $1.138 \mathrm{e}-4$ & 2.04 & $2.969 \mathrm{e}-2$ & 2.01 \\
$1.250 \mathrm{e}-2$ & $7.354 \mathrm{e}-6$ & 2.39 & $6.318 \mathrm{e}-5$ & 2.33 & $1.667 \mathrm{e}-2$ & 2.30 \\
$1.000 \mathrm{e}-2$ & $4.310 \mathrm{e}-6$ & 2.30 & $3.755 \mathrm{e}-5$ & 1.96 & $9.975 \mathrm{e} 03$ & 2.74 \\
$8.333 \mathrm{e}-3$ & $2.835 \mathrm{e}-6$ & - & $2.628 \mathrm{e}-5$ & - & $6.055 \mathrm{e}-3$ & - \\
\hline
\end{tabular}

TABLE 4. Convergence rates: nonlinear problem, biquadratic elements for $\mathbf{u}$, Raviart-Thomas space of order 1 for $p$ and $\mathbf{z}$.

Convergence rates are established by running cases for 6 levels of grid refinement, starting with $H=h=0.05$. The errors are calculated using the difference between the numerical solution and the exact solution, in the case of the linear problem (tables 1 and 2) and the difference between the numerical solution associated to each grid and the numerical solution associated to a much finer grid, in the case of the nonlinear problem (tables 3 and 4). 
The numerical results correspond to the solution after 1000 time iterations at time $t=6 e-5$.

In our experiments we used standard continuous bilinear elements to approximate the displacement and the lowest Raviart-Thomas space for the flow variables (tables 1 and 3), and standard continuous biquadratic linear elements to approximate the displacement and the Raviart-Thomas space of order 1 for the flow variables (tables 2 and 4 ).

The convergence rates conform the expected values.

\section{Conclusions}

The numerical solution of a fully coupled geomechanics and fluid-flow model for astress-sensitive reservoir problems was considered. The CG/mixed method for the nonlinear system produces optimal convergence rates with respect to regularity. A cut-off operator was introduced in the CG/mixed formulation to derive convergence. Numerical experiments support our convergence results. The theoretical error estimates we derived include the possibility for the displacement and the flow variables being calculated on different grids.

\section{References}

[1] Y. Abousleiman, A.H.-D. Cheng, L. Cui, E. Detournay, J.-C. Roegiers, Mandel's problem revisited, Géotechnique, v.46, n.2, 187195, 1996.

[2] S. Brenner, L. Scott, The mathematical theory of finite element methods, Second Edition, Texts in Applied Mathematics, 15. Springer-Verlag, New York, 1994.

[3] F. Brezzi, M. Fortin, Mixed and Hybrid Finite Element Methods, Springer Series In Computational Mathematics; v.15, 1991.

[4] L.Y. Chin, R. Raghavan, L.K. Thomas, Fully-coupled geomechanics and fluid-flow analysis of wells with stress-dependent permeability, SPE Journal, v.5, n.1, p.32-45, 2000.

[5] E. Emmrich, Stability and error of the variable two-step BDF for semilinear parabolic problems, J. Appl. Math. Comput. 19, n.1-2, 33-55, 2005.

[6] X. Gai. A coupled geomechanics and reservoir flow model on parallel computers. PhD thesis, The University of Texas at Austin, Austin, Texas, 2004.

[7] R. Liu. Discontinuous Galerkin finite element solution for poromechanics. PhD thesis, The University of Texas at Austin, Austin, Texas, 2004.

[8] J. Mandel, Consolidation des sols (étude mathématique), Géotechnique, v.3, 287299, 1953.

[9] J.C. Nedelec, Mixed finite elements in $\mathbb{R}^{3}$, Numerische Mathematik, v. 35, n.3, 315-341, 1980.

[10] P.J. Philips, Finite element methods for linear poroelasticity: theoritical and computational results, Ph.D. thesis, UT Austin, 2005.

[11] P.J. Phillips, M.F. Wheeler, A coupling of mixed and continuous Galerkin finite element methods for poroelasticity I: the continuous-in-time case, Computational Geosciences, v.11 n.2, p.131-144, 2007. 
[12] P.J. Phillips, Mary F. Wheeler, A coupling of mixed and continuous Galerkin finite element methods for poroelasticity II: the discrete-in-time case, Computational Geosciences, v.11 n.2, p.145-158, 2007.

[13] R. E. Showalter, Diffusion in Poro-Elastic Media, Journal of Mathematical Analysis and Applications, v. 251,p. 310-340, 2000.

[14] S. Sun, M.F. Wheeler, Discontinuous Galerkin methods for coupled flow and reactive transport problems, Applied Numerical Mathematics, v.52 n.2-3, p.273-298, 2005.

[15] R. Raghavan, L.Y. Chin, Productivity changes in reservoirs with stress-dependent permeability, SPE Reservoir evaluation \& engineering, v.7, n. 4, p. 308-315, 2004.

[16] P. A. Raviart, J.M. Thomas, A mixed finite element method for second order elliptic problems, Lecture Notes in Mathematics, Springer, v. 606, p. 292-315, 1977.

Sílvia BARBEIRo

CmUC, Department of Mathematics, University of Coimbra, Apartado 3008, 3001-454 CoImbra

The Institute for Computational Engineering and Sciences, The University of Texas at Austin, 1 University Station C0200 Austin, Texas 78712

E-mail address: silvia@mat.uc.pt

$U R L:$ http://www.mat.uc.pt/〜silvia

MARY F. WheELER

The Institute for Computational Engineering and Sciences, The University of Texas at Austin, 1 University Station C0200 Austin, Texas 78712

E-mail address: mfw@ices.utexas.edu

$U R L$ : http://users.ices.utexas. edu/ ${ }^{\sim} \mathrm{mf} w$ 